MACROSCOPIC ELECTROSTATIC STABILITY PROPERTIES OF

NONRELATIVISTIC NONNEUTRAL ELECTRON FLOW IN A

CYLINDRICAL DIODE WITH APPLIED MAGNETIC FIELD

Ronald C. Davidson

Kang Tsang

PFC/JA-83-29

July, 1983 


\title{
MACROSCOPIC ELECTROSTATIC STABILITY PROPERTIES OF NONRELATIVISTIC NONNEUTRAL ELECTRON FLOW IN A CYLINDRICAL DIODE WITH APPLIED MAGNETIC FIELD
}

\author{
Ronald C. Davidson \\ Plasma Fusion Center \\ Massachusetts Institute of Technology \\ Cambridge, Massachusetts 02139 \\ and \\ Kang Tsang \\ Science Applications, Inc. \\ Boulder, Colorado 80302
}

\begin{abstract}
Electrostatic stability properties of nonrelativistic nonneutral electron flow in a cylindrical diode with applied magnetic field $B_{0} \hat{e}_{z}$ are investigated within the framework of the macroscopic cold-fluid-Poisson equations. The electrostatic eigenvalue equation is derived for perturbations about the general class of slow rotationai cquilibria with angular velocity profile $\omega_{b}^{-}(r)=V_{\theta b}^{0}(r) / r=\left(\omega_{c} / 2\right)\left\{1-\left[1-\left(4 / \omega_{c}^{2} r^{2}\right) \int_{a}^{r} d r^{\prime} r^{\prime} \omega_{p b}^{2}\left(r^{r}\right)\right]^{1 / 2}\right\}$. Here, $\omega_{c}=e B_{0} / m c, \omega_{p b}^{2}(r)=4 x n_{b}^{0}(r) e^{2} / m, n_{b}^{0}(r)$ is the equilibrium electron density profile, the cathode is located at $r=a$ and the anode at $r=b$. Space-charge-limited flow is assumed with $E_{r}^{0}(r=a)=0$ and $\phi^{0}(r=a)=0$. The exact eigenvalue equation is simplified for low-frequency flute perturbations with $k_{z}=0$ and $\left|\omega-\ell \omega_{b}^{-}(r)\right|^{2} \ll \omega_{c}^{2}-\omega_{p b}^{2}(r)$, assuming $\omega_{p b}^{2}(r)<\omega_{c}^{2}$ and a moderate-aspect-ratio diode $\left(R_{0} \gg b-a\right)$. In this regime, it is shown that $\partial n_{b}^{0} / \partial r \leq 0$ over the interval $a \leq r \leq b$ is a sufficient condition for stability, and specific examples of stable oscillations (rectangular density profile) and weak resonant diocotron instability (gentle density bump) are analyzed in detail. Finally, the exact eigenvalue equation is solved numerically for a wide range of density profiles $n_{b}^{0}(r)$ and values of $\omega_{p b}^{2}(r) / \omega_{c}^{2}$ leading to weak and strong instability driven by velocity shear with $\partial \omega_{b}^{-}(r) / \partial r \neq 0$.
\end{abstract}




\section{INTRODUCTION AND SUMMARY}

The use of high-voltage diodes to generate intense charged particle beams for inertial confinement fusion applications ${ }^{1-4}$ has resulted in a concomitant need for a better theoretical understanding of the equilibrium and stability properties of nonneutral electron flow in various diode configurations. While there is a growing literature ${ }^{5-13}$ on the equilibrium and stability properties of nonneutral plasmas based on the Vlasov-Maxwell equations, it is often difficult in a kinetic treatment to obtain detailed estimates of instability growth rates, primarily because of the complications introduced by strong spatial inhomogeneities and intense self fields. Therefore, in the present analysis, we investigate the stability properties of nonneutral electron flow in a cylindrical diode making use of a macroscopic cold-fluid model ${ }^{14-20}$ for the electrons. The stability analysis is electrostatic and assumes nonrelativistic laminar electron flow. However, the present formalism can be extended in a relatively straightforward manner to include electromagnetic and relativistic effects. ${ }^{21}$

In a recent calculation, ${ }^{5}$ we made use of global conservation constraints satisfied by the fully nonlinear Vlasov-Maxwell equations to derive a sufficient condition for stability of the class of self-consistent planar diode equilibria $f_{b}^{0}\left(H, P_{y}\right)$. In the present analysis, we make use of a macroscopic, cold-fluid model (Secs. II and III) to investigate electrostatic stability properties of nonrelativistic sheared electron flow in a cylindrical diode with strong applied axial magnetic field $B_{0} \dot{e}_{2}$ (Fig. 1). After reviewing the cold-fluid equilibrium properties, the linearized fluid-Poisson equations (25)-(27) are used in Sec. III to investigate stability behavior for electrostatic perturbations about a nonneutral cylindrical equilibrium characterized by (general) electron density profile $n_{b}^{0}(r)$ and self-consistent azimuthal velocity profile $V_{\theta b}^{0}(r)$ defined by [Eq. (15)]

$$
V_{\theta b}^{0}(r)=\omega_{b}^{-}(r) r=\frac{r \omega_{c}}{2}\left\{1-\left[1-\frac{4}{\omega_{c}^{2} r^{2}} \int_{a}^{r} d r^{\prime} r^{\prime} \omega_{p b}^{2}\left(r^{\prime}\right)\right]^{1 / 2}\right\}
$$

Here, the cathode is located at $r=a$, and the anode at $r=b$ (Fig. 1). Moreover, $\omega_{c}=e B_{0} / m c$ is the (nonrelativistic) electron cyclotron frequency, and $\omega_{p b}^{2}(r)=4 \pi n_{b}^{0}(r) e^{2} / m$ is the electron plasma frequencysquared. For perturbations with complex oscillation frequency $\omega=\omega_{r}+i \gamma$, axial wavenumber $k_{z}$, and azimuthal harmonic number $\ell$ [Eq. (28)], the linearized fluid-Poisson equations (29)-(33) can be combined to give [Eq. (34)]

$$
\begin{gathered}
\frac{1}{r} \frac{\partial}{\partial r}\left\{r\left[1-\frac{\omega_{p b}^{2}}{\nu_{b}^{2}}\right] \frac{\partial}{\partial r} \delta \phi^{\ell}\right\}-\frac{\ell^{2}}{r^{2}}\left[1-\frac{\omega_{p b}^{2}}{\nu_{b}^{2}}\right] \delta \phi^{\ell}-k_{z}^{2}\left[1-\frac{\omega_{p b}^{2}}{\left(\omega-\ell \omega_{b}^{-}\right)^{2}}\right] \delta \phi^{\ell} \\
=-\frac{\ell \delta \phi^{\ell}}{r} \frac{1}{\left(\omega-\ell \omega_{b}^{-}\right)} \frac{\partial}{\partial r}\left[\frac{\omega_{p b}^{2}}{\nu_{b}^{2}}\left(2 \omega_{b}^{-}-\omega_{c}\right)\right] .
\end{gathered}
$$


Here, $\delta \phi^{e}\left(k_{z}, r\right)$ is the perturbed electrostatic potential, $\omega_{p b}^{2}(r)=4 \pi n_{b}^{0}(r) e^{2} / m$, and $\nu_{b}^{2}(r)$ is defined by [Eq. (38)]

$$
\nu_{b}^{2}(r)=\left[\omega-\ell \omega_{b}^{-}(r)\right]^{2}-\left[\omega_{c}^{2}-\omega_{p b}^{2}(r)-2 \omega_{c} \omega_{E}(r)\right],
$$

where $\omega_{E}(r)=-c E_{r}^{0}(r) / B_{0} r$ is given by [Eq. (37)]

$$
\omega_{E}(r)=\frac{1}{r^{2} \omega_{c}} \int_{a}^{r} d r^{\prime} r^{\prime} \omega_{p b}^{2}\left(r^{\prime}\right)
$$

The eigenvalue equation (34) provides an exact cold-fluid description of electrostatic stability properties, assuming nonrelativistic electron flow. For a moderate-aspect-ratio diode with $d \ll a$ (Fig. 1), it follows that $\omega_{E}(r) \ll \omega_{c}[E q .(39)]$. and the eigenvalue equation (34) further simplifies to give the approximate result [Eq. (43)]

$$
\begin{aligned}
\frac{1}{r} & \frac{\partial}{\partial r}\left\{r\left[1-\frac{\omega_{p b}^{2}}{\left(\omega-\ell \omega_{E}\right)^{2}-\left(\omega_{c}^{2}-\omega_{p b}^{2}\right)}\right] \frac{\partial}{\partial r} \delta \phi^{\ell}\right\} \\
& -\frac{\ell^{2}}{r^{2}}\left[1-\frac{\omega_{p b}^{2}}{\left(\omega-\ell \omega_{E}\right)^{2}-\left(\omega_{c}^{2}-\omega_{p b}^{2}\right)}\right] \delta \phi^{\ell}-k_{z}^{2}\left[1-\frac{\omega_{p b}^{2}}{\left(\omega-\ell \omega_{E}\right)^{2}}\right] \delta \phi^{\ell} \\
= & \frac{\ell \delta \phi^{\ell}}{r} \frac{\omega_{c}}{\left(\omega-\ell \omega_{E}\right)} \frac{\partial}{\partial r}\left[\frac{\omega_{p b}^{2}}{\left(\omega-\ell \omega_{E}\right)^{2}-\left(\omega_{c}^{2}-\omega_{p b}^{2}\right)}\right] .
\end{aligned}
$$

In Secs. IV and VI, we analyze Eq. (43) for the special case of low-frequency flute perturbations with [Eqs. (44) and (46)]

$$
k_{z}=0 \text { and }\left|\omega-\ell \omega_{E}(r)\right|^{2} \ll \omega_{c}^{2}-\omega_{p b}^{2}(r)
$$

assuming that the electron density is below the condition for Brillouin flow, i.e., $\omega_{p b}^{2}(r)<\omega_{c}^{2}$. In this case, the eigenvaluc equation (43) can be approximated by [Eq. (47)]

$$
\begin{aligned}
& \frac{1}{r} \frac{\partial}{\partial r}\left\{r\left[1+\frac{\omega_{p b}^{2}(r)}{\omega_{c}^{2}-\omega_{p b}^{2}(r)}\right] \frac{\partial}{\partial r} \delta \phi^{\ell}\right\}-\frac{\ell^{2}}{r^{2}}\left[1+\frac{\omega_{p b}^{2}(r)}{\omega_{c}^{2}-\omega_{p b}^{2}(r)}\right] \delta \phi^{\ell} \\
& \quad=-\frac{\ell \delta \phi^{\ell}}{r} \frac{\omega_{c}}{\left[\omega-\ell \omega_{E}(r)\right]} \frac{\omega_{c}^{2}}{\left[\omega_{c}^{2}-\omega_{p b}^{2}(r)\right]^{2}} \frac{\partial}{\partial r} \omega_{p b}^{2}(r) .
\end{aligned}
$$

In Sec. IV.A, we make direct use of the eigenvalue equation (47) to show that 


$$
\frac{\partial}{\partial r} \omega_{p b}^{2}(r) \leq 0
$$

over the interval $a \leq r \leq b$ is a sufficient condition to assure electrostatic stability [Eq. (55)]. That is, equilibrium density profiles that decrease monotonically from the cathode to the anode are electrostatically stable. For the special case of weak resonant diocotron instability with growth rate $\gamma=I m \omega \ll\left|\omega_{r}\right|$, a formal expression for the growth rate $\gamma$ is derived in Sec. IV.B [Eq. (60)],

$$
\begin{aligned}
\gamma=-\frac{D_{i}\left(\omega_{r}\right)}{\partial D_{r} / \partial \omega_{r}}=- & \pi \int_{a}^{b} d r\left|\delta \phi^{\ell}\right|^{2} \frac{\omega_{c}^{3} \delta\left[\omega_{r}-\ell \omega_{E}(r)\right]}{\left[\omega_{c}^{2}-\omega_{p b}^{2}(r)\right]^{2}} \frac{\partial}{\partial r} \omega_{p b}^{2}(r) \\
& \times\left[\int_{a}^{b} d r\left|\delta \phi^{\ell}\right|^{2} \frac{\omega_{c} P}{\left[\omega_{r}-\ell \omega_{E}(r)\right]^{2}} \frac{\omega_{c}^{2}}{\left[\omega_{c}^{2}-\omega_{p b}^{2}(r)\right]^{2}} \frac{\delta}{\partial r} \omega_{p b}^{2}(r)\right]^{-1},
\end{aligned}
$$

where $\omega_{r}=$ Rew is determined from the dispersion relation (59). In Sec. VI, analytic solutions to the electrostatic eigenvalue equation (47) are determined both for stable surface modes on an annular electron beam (Sec. VI.A), and for weak resonant diocotron instability driven by a small density bump with $\partial \omega_{p b}^{2} /\left.\theta_{r}\right|_{r=r_{0}}>0$ (Sec. VI.B).

Because of the very general nature of the stability theorem obtained directly from the eigenvalue equation (47), we have also developed an indirect proof that $\delta n_{b}^{0} / \partial r \leq 0$ is a sufficient condition for stability (Sec. V). The analysis in Sec. $V$ is based on a cold-fluid guiding-center model in which electron inertial effects are neglected $\left(m \rightarrow 0\right.$ and $B_{0} \rightarrow \infty$ ) and the motion of an electron fluid element is determined from $V_{b}=$ $\left(-c / B_{0}\right) \nabla \phi \times \hat{e}_{z}$ [Eq. (64)]. Making use of the continuity equation for $n_{b}(r, \theta, t)$ [Eq. (66)] and Poisson's equation for $\phi(r, \theta, t)$ [Eq. (67)], it is found that [Eq. (76)]

$$
\Delta U_{G}=\int d^{2} r\left[G\left(n_{b}\right)-G\left(n_{b}^{0}\right)\right]=\text { const, }
$$

and [Eq. (84)]

$$
\Delta U_{r}=\int d^{2} r r^{2}\left(n_{b}-n_{b}^{0}\right)=\text { const, }
$$

are exact global conservation constraints, where $\int d^{2} r=\int_{0}^{2 \pi} d \theta \int_{a}^{b} d r r$. Note that Eq. (84) corresponds to conservation of density-weighted average radial guiding center location. Defining the effective free energy $\Delta F=\Delta U_{r}+\Delta U_{G}[$ [Eq. (85)], it is shown in Sec. V that [Eq. (91)]

$$
\frac{\partial n_{b}^{0}}{\partial r} \leq 0
$$


over the interval $a \leq r \leq b$ is a sufficient condition for electrostatic stability to small-amplitude perturbations. We reiterate that the indirect proof of this very powerful stability theorem in Sec. $V$ has been based on a coldfluid guiding-center model with $m \rightarrow 0$ and $B_{0} \rightarrow \infty$.

Finally, to conclude this paper, in Sec. VII we solve numerically the exact eiectrostatic eigenvalue equation (34) for a wide range of electron density profiles $n_{b}^{0}(r)$ leading to weak and strong instability driven by velocity shear. 


\section{LAMINAR COLD-FLUID EQUILIBRIUM FOR A CYLINDRICAL DIODE}

\section{A. Theoretical Model and Assumptions}

We consider here the equilibrium properties $(\partial / \partial t=0)$ for a cold, nonneutral pure electron plasma confined in the cylindrical diode configuration illustrated in Fig. 1. The cathode is located at $r=a$ and the anode at $r=b$, where $d=b-a$ is the anode-cathode spacing. In addition, the electron fluid is immersed in a uniform applied magnetic field $B_{0} \hat{e}_{z}$. The equilibrium analysis is based on a macroscopic cold-fluid description with the following simplifying assumptions:

(a) The electron fluid is uniform in the $z$-direction, with $\partial n_{b}^{0}(x) / \partial z=0$ and $\partial \nu_{b}^{0}(x) / \partial z=0$, and there is no equilibrium electric ficld parallel to $B_{0} \hat{e}_{z}$, i.e., $E^{0}(x) \cdot \hat{e}_{z}=0$.

(b) The equilibrium radial density profile and the azimuthal flow velocity profile are assumed to be azimuthally symmetric, i.e.,

$$
\begin{aligned}
& n_{b}^{0}(x)=n_{b}^{0}(r), \\
& V_{b}^{0}(x)=V_{\theta b}^{0}(r) \hat{e}_{\theta,}
\end{aligned}
$$

where $r$ is the radial distance from the axis of symmetry, and $\hat{e}_{\theta}$ and $\hat{e}_{z}$ are unit vectors in the $\theta-$ and $z-$ directions, respectively. The equilibrium continuity equation $\nabla \cdot\left[n_{b}^{0}(x) \nu_{b}^{0}(x)\right]=0$ is automatically satisfied for general profiles $n_{b}^{0}(r)$ and $V_{\theta b}^{0}(r)$.

(c) The azimuthal current $J_{\theta}^{0}(r)=-e n_{b}^{0}(r) V_{\theta b}^{0}(r)$ will generally induce an axial self-magnetic ficld $B_{z}^{a}(r)$. Throughout the present analysis, it is assumed that the azimuthal current is sufficiently weak that the axial selfmagnetic ficld is negligibly small in comparison with the applied magnetic field, i.e.,

$$
\left|B_{z}^{s}(r)\right| \ll B_{0}
$$

(d) The electron fluid is assumed to be sufficiently cold that pressure gradients can be neglected in the equilibrium force balance equation, i.e.,

$$
\frac{\partial}{\partial x} \cdot P_{b}^{0}=0
$$

It is convenient to introduce the notation

$$
V_{\theta b}^{0}(r)=\omega_{b}(r) r,
$$


in the subsequent analysis. Within the context of Assumptions (a)-(c), the equilibrium field components are

$$
\begin{aligned}
& E_{0}(x)=E_{r}^{0}(r) \hat{e}_{r} \\
& B_{0}(x)=B_{0} \hat{e}_{r,}
\end{aligned}
$$

where the electric field is determined from the steady-state Poisson equation

$$
\frac{1}{r} \frac{\partial}{\partial r}\left[r E_{r}^{0}(r)\right]=-4 \pi e n_{b}^{0}(r),
$$

and $-e$ is the electron charge. Integrating Eq. (6) gives the equilibrium radial electric field

$$
E_{r}^{0}(r)=-\frac{4 \pi e}{r} \int_{a}^{r} d r^{\prime} r_{b}^{0}(r),
$$

where space-charge-limited flow with

$$
E_{r}^{0}(r=a)=0
$$

is assumed. We introduce the electrostatic potential $\phi_{0}(r)$, where $E_{r}^{0}=-\partial \phi^{0} / \partial r$, and impose the boundary conditions

$$
\begin{aligned}
& \phi_{0}(r=a)=0, \\
& \phi_{0}(r=b)=V .
\end{aligned}
$$

The anode voltage $V$ consistent with Eqs. (7) and (8) is given by

$$
V=\phi_{0}(b)=4 \pi e \int_{a}^{b} \frac{d r^{\prime \prime}}{r^{\prime \prime}} \int_{a}^{r^{\prime \prime}} d r^{\prime} r^{\prime} n_{b}^{0}(r) .
$$

(e) Finally, for analytic simplicity, it is assumed in the present analysis that the fluid motion is nonrelativistic. In equilibrium, radial force balance on a fluid element can therefore be expressed as

$$
\frac{-m V_{\theta b}^{02}(r)}{r}=-e\left[E_{r}^{0}(r)+\frac{1}{c} V_{\theta b}^{0}(r) B_{0}\right] .
$$




\section{B. Equilibrium Flow Properties}

Substituting Eqs. (4) and (7) into Eq. (11) gives (for $a \leq r \leq b$ )

$$
\omega_{b}^{2}(r)-\omega_{b}(r) \omega_{c}+\frac{1}{r^{2}} \int_{a}^{r} d r^{\prime} r^{\prime} \omega_{p b}^{2}\left(r^{\prime}\right)=0,
$$

where

$$
\omega_{c}=\frac{e B_{0}}{m c} \quad \text { and } \quad \omega_{p b}^{2}(r)=\frac{4 \pi e^{2} n_{b}^{0}(r)}{m}
$$

are the (nonrelativistic) cyclotron frequency and plasma frequency-squared. Solving Eq. (12) for the angular velocity profile $\omega_{b}(r)$ gives

$$
\omega_{b}(r)=\omega_{b}^{ \pm}(r)=\frac{\omega_{c}}{2}\left\{1 \pm\left[1-\frac{4}{\omega_{c}^{2} r^{2}} \int_{a}^{r} d r^{\prime} r^{\prime} \omega_{p b}^{2}\left(r^{\prime}\right)\right]^{1 / 2}\right\} .
$$

Note from Eq. (14) that there are $t w o$ allowed equilibrium rotation frequencies, with $\omega_{b}^{+}(r)$ corresponding to a fast rotational equilibrium, and $\omega_{b}^{-}(r)$ corresponding to a slow rotational equilibrium. Note also from Eq. (14) that $\omega_{b}^{-}(r=a)=0$ at the cathode, whereas $\omega_{b}^{+}(r=a)=\omega_{c}$. In the subsequent equilibrium and stability analysis, it is assumed that

$$
V_{\theta b}^{0}(r)=\omega_{b}^{-}(r) r=\frac{r \omega_{c}}{2}\left\{1-\left[1-\frac{4}{\omega_{c}^{2} r^{2}} \int_{a}^{r} d r^{\prime} r^{\prime} \omega_{p b}^{2}(r)\right]^{1 / 2}\right\},
$$

corresponding to a slow rotational equilibrium. In the special case where

$$
\frac{4}{r^{2} \omega_{c}^{2}} \int_{a}^{r} d r^{\prime} r^{\prime} \omega_{p b}^{2}\left(r^{\prime}\right) \ll 1
$$

Eq. (15) can be approximated by

$$
V_{\theta b}^{0}(r)=\frac{1}{\omega_{c} r} \int_{a}^{r} d r^{\prime} r^{\prime} \omega_{p b}^{2}\left(r^{\prime}\right)=-\frac{c E_{r}^{0}(r)}{B_{0}},
$$

corresponding to an $E_{0}(x) \times B_{0} \hat{e}_{z}$ equilibrium rotation of a fluid element. In general, however, centrifugal effects should also be retained in Eq. (11), which gives the expression for $V_{\theta b}^{0}(r)$ in Eq. (14).

From Eq. (15), $\omega_{b}^{-}(r)$ can be determined for a broad class of equilibrium density profiles $n_{b}^{0}(r)$. By way of illustration, consider the rectangular density profile [Fig. 2(a)] specified by

$$
n_{b}^{0}(r)= \begin{cases}\hat{n}_{b}=\text { const., } & a \leq r<r_{b}, \\ 0, & r_{b}<r \leq b,\end{cases}
$$


Substituting Eq. (18) into Eq. (15) gives [Fig. 2(b)]

$$
\omega_{b}^{-}(r)=\frac{\omega_{c}}{2}\left\{1-\left[1-\frac{2 \hat{\omega}_{p b}^{2}}{\omega_{c}^{2}}\left(1-\frac{a^{2}}{r^{2}}\right)\right]^{1 / 2}\right\}
$$

for $a \leq r \leq r_{b}$. Here $\hat{\omega}_{p b}^{2}=4 \pi \hat{n}_{b} e^{2} / m=$ const. Note that $\omega_{b}^{-}(r)$ assumes its maximum value at $r=r_{b}$. For $a=0$, it follows from Eq. (19) that $\omega_{b}^{-}=$const. and $\partial \omega_{b}^{-} / \partial r=0$. For $a \neq 0$, however, there is generally $a$ shear in the angular velocity profile with $\partial \omega_{b}^{-}(r) / \partial r \neq 0$.

Finally, an important frequency known as the vortex frequency, $\omega_{b v}$, enters the subsequent stability analysis. Here, $\omega_{b v}$ is defined by

$$
\omega_{b v}(r) \equiv \omega_{b}^{+}(r)-\omega_{b}^{-}(r)= \pm\left[2 \omega_{b}^{t}(r)-\omega_{c}\right]
$$

From Eq. (14), we find that

$$
\left(\omega_{b}^{+}-\omega_{b}^{-}\right)^{2}=\omega_{c}^{2}-\frac{4}{r^{2}} \int_{a}^{r} d r^{\prime} r^{\prime} \omega_{p b}^{2}(r) .
$$

Moreover, $\omega_{p b}^{2}(r)$ can be expressed directly in terms of $\left(\omega_{b}^{+}-\omega_{b}^{-}\right)^{2}$ by

$$
\omega_{p b}^{2}(r)=-\frac{1}{4 r} \frac{\partial}{\partial r}\left[r^{2}\left(\omega_{b}^{+}-\omega_{b}^{-}\right)^{2}\right]
$$




\section{ELECTROSTATICEIGENVALUEEQUATION}

\section{FOR NONRELATIVISTICFI.OW IN A CYLINDRICALDIODE}

In this section, we derive the eigenvalue equation for small-amplitude electrostatic perturbations about the general class in laminar cold-fluid equilibria described in Sec. II. Assuming $\delta B(x, t) \simeq 0$ and

$$
E(x, t)=-\nabla \phi(x, t),
$$

each quantity of physical interest is expressed as its equilibrium value plus a perturbation. That is,

$$
\begin{aligned}
& n_{b}(x, t)=n_{b}^{0}(r)+\delta n_{b}(x, t), \\
& V_{b}(x, t)=V_{\theta b}^{0}(r) \hat{e}_{b}+\delta V_{b}(x, t), \\
& E(x, t)=E_{r}^{0}(r) \hat{e}_{r}-\nabla \delta \phi(x, t), \\
& B(x, t)=B_{0} \hat{e}_{z,}
\end{aligned}
$$

where $\hat{e}_{r}, \hat{e}_{\theta}$, and $\hat{e}_{z}$ are unit vectors in the $r-, \theta-$, and $z$-directions, respectively. For small-amplitude perturbations, the evolution of $\delta n_{b}(x, t), \delta V_{b}(x, t)$ and $\delta \phi(x, t)$ is determined from the macroscopic cold-fluid-Poisson equations

$$
\begin{gathered}
0=\frac{\partial}{\partial t} \delta n_{b}(x, t)+\nabla \cdot\left[n_{b}^{0}(r) \delta V_{b}(x, t)+\delta n_{b}(x, t) V_{\theta b}^{0}(r) \hat{e}_{\theta}\right] \\
\frac{\partial}{\partial t} \delta V_{b}(x, t)+V_{\theta b}^{0}(r) \hat{e}_{\theta} \cdot \nabla \delta V_{b}(x, t)+\delta V_{b}(x, t) \cdot \nabla\left[V_{\theta b}^{0}(r) \hat{e}_{\theta}\right] \\
=\frac{-e}{m}\left[-\nabla \delta \phi(x, t)+\frac{\delta V_{b}(x, t) \times B_{0} \dot{e}_{z}}{c}\right] \\
\nabla^{2} \delta \phi(x, t)=4 \pi e \delta n_{b}(x, t) .
\end{gathered}
$$

To determine the stability properties for perturbations about equilibrium, a normal-mode approach is adopted. It is assumed that the time variation of perturbed quantities is of the form $\exp (-i \omega t)$, where the complex oscillation frequency $\omega$ is determined consistently from Eqs. (25)-(27). If $\operatorname{Im} \omega>0$, then the perturbations grow and the equilibrium configuration is unstable. In analyzing Eqs. (25)-(27), the perturbations are assumed to be spatially periodic in the $z$-direction. The $\theta$ - and $z$-dependences of all perturbed quantities are Fourier decomposed according to 


$$
\delta \psi(r, \theta, z, t)=\sum_{\ell=-\infty}^{\infty} \sum_{k_{z}=-\infty}^{\infty} \delta \psi^{\ell}\left(r, k_{z}\right) \exp \left\{i\left(\ell \theta+k_{z} z-\omega t\right)\right\}
$$

Substituting Eq. (28) into Eqs. (25)-(27), it can be shown that the Fourier amplitudes $\delta n_{b}^{\ell}\left(r, k_{z}\right), \delta V_{r b}^{\ell}\left(r, k_{z}\right)$, etc., satisfy

$$
\begin{gathered}
-i\left(\omega-\ell \omega_{b}^{-}\right) \delta n_{b}^{\ell}+\frac{1}{r} \frac{\partial}{\partial r}\left(r n_{b}^{0} \delta V_{r b}^{\ell}\right)+\frac{i l n_{b}^{0} \delta V_{\theta b}^{\ell}}{r}+i k_{z} n_{b}^{0} \delta V_{z b}^{\ell}=0, \\
-i\left(\omega-\ell \omega_{b}^{-}\right) \delta V_{r b}^{\ell}-\left(-\omega_{c}+2 \omega_{b}^{-}\right) \delta V_{\theta b}^{\ell}=\frac{e}{m} \frac{\partial}{\partial r} \delta \phi^{\ell}, \\
-i\left(\omega-\ell \omega_{b}^{-}\right) \delta V_{\theta b}^{\ell}+\left[-\omega_{c}+\frac{1}{r} \frac{\partial}{\partial r}\left(r^{2} \omega_{b}^{-}\right)\right] \delta V_{r b}^{\ell}=\frac{e}{m} \frac{i \ell \delta \phi^{\ell}}{r}, \\
-i\left(\omega-\ell \omega_{b}^{-}\right) \delta V_{z b}^{\ell}=\frac{e}{m} i k_{z} \delta \phi^{\ell}, \\
\frac{1}{r} \frac{\partial}{\partial r} r \frac{\delta}{\partial r} \delta \phi^{\ell}-\frac{\ell^{2}}{r 2} \delta \phi^{\ell}-k_{z}^{2} \delta \phi^{\ell}=4 \pi e \delta n_{b}^{\ell},
\end{gathered}
$$

where $-e$ is the electron charge, $\omega_{c}=e B_{0} / m c$ is the cyclotron frequency, and a slow rotational equilibrium with $\omega_{b}(r)=\omega_{b}^{-}(r)=V_{\theta b}^{0}(r) / r$ is assumed in Eqs. (29)-(33). The equilibrium angular velocity $\omega_{b}^{-}(r)$ in Eqs. (29) $-(33)$ is related to the equilibrium density profile $n_{b}^{0}(r)$ by Eq. (15). The perturbations in density and mean fluid velocities in Fqs. (29)-(32) can be eliminated in favor of $\delta \phi^{\ell}\left(r, k_{z}\right)$. Poisson's cquation for the perturbed electrostatic potential can then be expressed in the form

$$
\begin{aligned}
\frac{1}{r} & \frac{\partial}{\partial r}\left\{r\left[1-\frac{\omega_{p b}^{2}}{\nu_{b}^{2}}\right] \frac{\partial}{\partial r} \delta \phi^{\ell}\right\}-\frac{\ell^{2}}{r^{2}}\left[1-\frac{\omega_{p b}^{2}}{\nu_{b}^{2}}\right] \delta \phi^{\ell}-k_{z}^{2}\left[1-\frac{\omega_{p b}^{2}}{\left(\omega-\ell \omega_{b}^{-}\right)^{2}}\right] \delta \phi^{\ell} \\
& =-\frac{\ell \delta \phi^{\ell}}{r} \frac{1}{\left(\omega-\ell \omega_{b}^{-}\right)} \frac{\partial}{\partial r}\left[\frac{\omega_{p b}^{2}}{\nu_{b}^{2}}\left(2 \omega_{b}^{-}-\omega_{c}\right)\right]
\end{aligned}
$$

where $\omega_{p b}^{2}(r)=4 \pi n_{b}^{0}(r) e^{2} / m$, and $\nu_{b}^{2}(r)$ is defined by

$$
\nu_{b}^{2}(r)=\left(\omega-\ell \omega_{b}^{-}\right)^{2}-\left(2 \omega_{b}^{-}-\omega_{c}\right)\left[\frac{1}{r} \frac{\partial}{\partial r}\left(r^{2} \omega_{b}^{-}\right)-\omega_{c}\right] .
$$


Equation (34) is valid for arbitrary $\omega_{p b}^{2}(r)$ and $\omega_{b}^{-}(r)$ consistent with Eq. (15). Operationally the procedure is to solve Eq. (34) for $\delta \phi^{\ell}\left(r, k_{z}\right)$ and $\omega$ as an eigenvalue problem. The solution to Eq. (34) is accessible analytically for certain simple density profiles.

Making use of the definition of $\omega_{b}^{-}(r)$ in Eq. (15), the quantity $\nu_{b}^{2}(r)$ defined in Eq. (35) can be expressed in the equivalent form

$$
\nu_{b}^{2}(r)=\left(\omega-\ell \omega_{b}^{-}\right)^{2}-\left[\omega_{c}^{2}-\omega_{p}^{2}(r)-\frac{2}{r^{2}} \int_{a}^{r} d r^{\prime} r^{\prime} \omega_{p}^{2}(r)\right]
$$

It should be emphasized that the $\omega_{p}^{2}(r)$ contributions in Eq. (36) arise from equilibrium space-charge effects associated with $E_{r}^{0}(r) \neq 0$. It should also be noted that for a thin annulus of electrons (large-aspect-ratio diode) the final term in Eq. (36) is typically small in comparison with $\omega_{p}^{2}(r)$. Defining

$$
\omega_{E}(r) \equiv-\frac{c E_{r}^{0}(r)}{B_{0} r}=\frac{1}{r^{2} \omega_{c}} \int_{a}^{r} d r^{\prime} r^{\prime} \omega_{p b}^{2}(r)
$$

the quantity $\nu_{b}^{2}(r)$ in Eq. (36) can also be expressed exactly as

$$
\nu_{b}^{2}(r)=\left(\omega-\ell \omega_{b}^{-}\right)^{2}-\left[\omega_{c}^{2}-\omega_{p b}^{2}(r)-2 \omega_{c} \omega_{E}(r)\right]
$$

Thus far, the electrostatic eigenvalue equation (34) is completely general. We now simplify Eq. (34) for the case of a cylindrical diode with moderately large aspect ratio $\left(R_{0} \gg d\right.$ in Fig. 1). In particular, it is assumed that

$$
\omega_{E}(r)=\frac{1}{\omega_{c} r^{2}} \int_{a}^{r} d r^{\prime} r^{\prime} \omega_{p b}^{2}\left(r^{\prime}\right) \ll \omega_{c}
$$

over the radial extent of the electron plasma. Note that Eq. (39) does not require that the electron density be low with $\omega_{p b}^{2}(r) \ll \omega_{c}^{2}$. Rather, evaluating Eq. (39) at $r=b$, the inequality in Eq. (39) is satisfied whenever

$$
\frac{\hat{\omega}_{p b}^{2}}{\omega_{c}^{2}} \frac{\Delta}{R_{0}} \ll 1,
$$

where $\hat{\omega}_{p b}^{2}=4 \pi \hat{n}_{b} e^{2} / m, \hat{n}_{b}$ is the characteristic (average) electron density, and $\Delta\left(\ll R_{0}\right)$ is the characteristic radial width of the electron density profile. Making use of Eq. (39), it follows from Eqs. (15) and (38) that $\omega_{b}^{-}(r)$ and $\nu_{b}^{2}(r)$ can be approximated by

$$
\omega_{b}^{-}(r)=\omega_{E}(r)
$$

and 


$$
\nu_{b}^{2}(r)=\left[\omega-\ell \omega_{E}(r)\right]^{2}-\left[\omega_{c}^{2}-\omega_{p b}^{2}(r)\right] .
$$

Moreover, within the context of Eqs. (39), (41) and (42), the electrostatic eigenvalue equation (34) can be approximated by

$$
\begin{aligned}
\frac{1}{r} & \frac{\partial}{\partial r}\left\{r\left[1-\frac{\omega_{p b}^{2}}{\left(\omega-\ell \omega_{E}\right)^{2}-\left(\omega_{c}^{2}-\omega_{p b}^{2}\right)}\right] \frac{\partial}{\partial r} \delta \phi^{\ell}\right\} \\
& -\frac{\ell^{2}}{r^{2}}\left[1-\frac{\omega_{p b}^{2}}{\left(\omega-\ell \omega_{E}\right)^{2}-\left(\omega_{c}^{2}-\omega_{p b}^{2}\right)}\right] \delta \phi^{\ell}-k_{z}^{2}\left[1-\frac{\omega_{p b}^{2}}{\left(\omega-\ell \omega_{E}\right)^{2}}\right] \delta \phi^{\ell} \\
= & \frac{\ell \delta \phi^{\ell}}{r} \frac{\omega_{c}}{\left(\omega-\ell \omega_{E}\right)} \frac{\partial}{\partial r}\left[\frac{\omega_{p b}^{2}}{\left(\omega-\ell \omega_{E}\right)^{2}-\left(\omega_{c}^{2}-\omega_{p b}^{2}\right)}\right]
\end{aligned}
$$

where $\omega_{p b}^{2}(r)=4 \pi n_{b}^{0}(r) e^{2} / m$, and $\omega_{E}(r)$ is defined in Eq. (37). 


\section{STABILITY THEOREM FOR LOW-FREQUENCY FLUTE PERTURBATIONS}

\section{A. Sufficient Condition for Stability}

The electrostatic eigenvalue equation (43) can be solved numerically for the eigenfunction $\delta \phi^{\ell}$ and the eigenfrequency $\omega$ for a broad range of electron density profiles $n_{b}^{0}(r)$. and specific numerical examples are presented in Sec. VII. In this section, we make use of Eq. (43) to determine a sufficient condition for $n_{b}^{0}(r)$ to be stable for low-frequency flute perturbations with

$$
k_{2}=0 \text {. }
$$

In particular, it is assumed that the electron density is below the condition for Brillouin flow

$$
\omega_{p b}^{2}(r)<\omega_{c}^{2} .
$$

[Note that $\omega_{p b}^{2}(r) \ll \omega_{c}^{2}$ is not required for proof of the stability theorem later in this section.] The eigenvalue equation (43) gencrally supports both high-frequency and low-frequency solutions. For present purposes, we examine Eq. (43) for low-frequency perturbations satisfying

$$
\left|\omega-\ell \omega_{E}(r)\right|^{2} \ll \omega_{c}^{2}-\omega_{p b}^{2}(r) .
$$

Making use of Eqs. (44) and (46), the eigenvalue equation (43) can be approximated by

$$
\begin{aligned}
& \frac{1}{r} \frac{\partial}{\partial r}\left\{r\left[1+\frac{\omega_{p b}^{2}(r)}{\omega_{c}^{2}-\omega_{p b}^{2}(r)}\right] \frac{\partial}{\partial r} \delta \phi^{\ell}\right\}-\frac{\ell^{2}}{r^{2}}\left[1+\frac{\omega_{p b}^{2}(r)}{\omega_{c}^{2}-\omega_{p b}^{2}(r)}\right] \delta \phi^{\ell} \\
& \quad=-\frac{\ell \delta \phi^{\ell}}{r} \frac{\omega_{c}}{\left[\omega-\ell \omega_{E}(r)\right]} \frac{\omega_{c}^{2}}{\left[\omega_{c}^{2}-\omega_{p b}^{2}(r)\right]^{2}} \frac{\partial}{\partial r} \omega_{p b}^{2}(r) .
\end{aligned}
$$

In Eq. (47), note that

$$
\epsilon_{\perp}(r)=1+\frac{\omega_{p b}^{2}(r)}{\omega_{c}^{2}-\omega_{p b}^{2}(r)}
$$

is the effective perpendicular dielectric function, and

$$
\omega=\omega_{r}+i \gamma
$$


is the complex eigenfrequency, with $\gamma=I m \omega>0$ corresponding to instability. Moreover, the boundary conditions used in solving Eq. (47) are

$$
\delta \phi^{\ell}(r=a)=0=\delta \phi^{\ell}(r=b)
$$

which assures that the tangential electric field $\delta E_{\theta}^{\ell}=-i \ell \delta \phi^{\ell}$ is equal to zero at the perfect conducting cathode $(r=a)$ and anode $(r=b)$.

To determine a sufficient condition for stability, we multiply Eq. (47) by $r \delta \phi^{\ell^{*}}$ and integrate from $r=a$ to $r=b$. This gives

$$
\begin{aligned}
0=D(\omega)= & \int_{a}^{b} d r r\left\{\left[\left|\frac{\partial}{\partial r} \delta \phi^{\ell}\right|^{2}+\frac{\ell^{2}}{r^{2}}\left|\delta \phi^{\ell}\right|^{2}\right]\left[1+\frac{\omega_{p b}^{2}(r)}{\omega_{c}^{2}-\omega_{p b}^{2}(r)}\right]\right. \\
& \left.-\frac{\ell\left|\delta \phi^{\ell}\right|^{2}}{r} \frac{\omega_{c}}{\left[\omega-\ell \omega_{E}(r)\right]} \frac{\omega_{c}^{2}}{\left[\omega_{c}^{2}-\omega_{p b}^{2}(r)\right]^{2}} \frac{\partial}{\partial r} \omega_{p b}^{2}(r)\right\}
\end{aligned}
$$

Expressing

$$
\frac{1}{\omega_{r}-\ell \omega_{E}+i \gamma}=\frac{\left(\omega_{r}-\ell \omega_{E}\right)-i \gamma}{\left(\omega_{r}-\ell \omega_{E}\right)^{2}+\gamma^{2}}
$$

we equate the real and imaginary parts of Eq. (51) separately to zero. The condition $D_{r}=R e D(\omega)=0$ gives

$$
\begin{aligned}
0=R e D(\omega)=\int_{a}^{b} d r r & \left\{\left|\frac{\partial}{\partial r} \delta \phi^{\ell}\right|^{2}+\frac{\ell^{2}}{r^{2}}\left|\delta \phi^{\ell}\right|^{2}\right]\left[1+\frac{\omega_{p b}^{2}}{\omega_{c}^{2}-\omega_{p b}^{2}(r)}\right] \\
& \left.-\frac{\ell\left|\delta \phi^{\ell}\right|^{2}}{r} \frac{\left.\omega_{c} \mid \omega_{r}-\ell \omega_{E}(r)\right]}{\left[\omega_{r}-\ell \omega_{E}(r)\right]^{2}+\gamma^{2}} \frac{\omega_{c}^{2}}{\left[\omega_{c}^{2}-\omega_{p b}^{2}(r)\right]^{2}} \frac{\partial}{\partial r} \omega_{p b}^{2}(r)\right\}
\end{aligned}
$$

whereas $D_{i}=\operatorname{ImD}(\omega)=0$ gives

$$
0=\operatorname{ImD}(\omega)=\ell \gamma \int_{a}^{b} d r\left|\delta \phi^{\ell}\right|^{2} \frac{\omega_{c}}{\left[\omega_{r}-\ell \omega_{E}(r)\right]^{2}+\gamma^{2}} \frac{\omega_{c}^{2}}{\left[\omega_{c}^{2}-\omega_{p b}^{2}(r)\right]} \frac{\partial}{\delta r} \omega_{p b}^{2}(r) .
$$

A sufficient condition for electrostatic stability follows from $\mathrm{Eq}$. (54). Let us assume that the density profile is monotonic decreasing with

$$
\frac{\theta}{\partial r} \omega_{p b}^{2}(r) \leq 0
$$


over the interval $a \leq r \leq b$, and that the system is unstable with $\gamma=I m \omega>0$. From Eq. (55), it follows that the integral in Eq. (54) is non-zero. Therefore, our assumption of instability $(\gamma>0)$ is incorrect for monotonic decreasing density profiles, and it necessarily follows that the system is stable whenever Eq. (55) is satisfied over the interval $a \leq r \leq b$. That is, Eq. (55) corresponds to a sufficient condition for stability. Expressed another way, for instability to exist it is necessary that $\partial \omega_{p b}^{2} / \partial r$ change sign in the interval $a \leq r \leq b$, or equivalently that

$$
\frac{1}{r} \frac{\partial}{\partial r}\left[r^{2} \omega_{E}(r)\right]
$$

change sign in the interval $a \leq r \leq b$, corresponding to a shear in the angular velocity profile.

We emphasize the powerful nature of the stability theorem in Eq. (55). For low-frequency flute perturbations satisfying Eqs. (44)-(46), all monotonic decreasing density profiles with $\partial \omega_{p b}^{2} / \partial r \leq 0$ are electrostatically stable. This stability condition has been derived without specifying the functional form of $n_{b}^{0}(r)$ or solving explicitly for the eigenfunction $\delta \phi^{\ell}(r)$. Equation (55) represents an important generalization of the stability theorem first derived by Briggs et al. ${ }^{19}$ for the case $\omega_{p}^{2}(r) \ll \omega_{c}^{2}$, i.e., $\epsilon_{\perp}(r)=1$.

\section{B. Growth Rate for Weak Resonant Instability}

From Eq. (51), it is straightforward to derive a formal expression for the growth rate $\gamma$ in circumstances where the instability growth rate $\gamma=I m \omega$ is weak with

$$
|\gamma| \ll\left|\omega_{r}\right|
$$

In particular, for small $\gamma$, we express

$$
D\left(\omega_{r}+i \gamma\right)=D_{r}\left(\omega_{r}\right)+i\left[D_{i}\left(\omega_{r}\right)+\gamma \frac{\partial D_{r}}{\partial \omega_{r}}\right]+\cdots
$$

and make use of

$$
\lim _{\gamma \rightarrow 0_{+}} \frac{1}{\omega_{r}-\ell \omega_{E}+i \gamma}=\frac{P}{\omega_{r}-\ell \omega_{E}}-i \pi \delta\left[\omega_{r}-\ell \omega_{E}(r)\right]
$$

where $P$ denotes Cauchy principal value. Substituting Eqs. (57) and (58) into Eq. (51) and setting real and imaginary parts equal to zero gives 


$$
\begin{aligned}
0=\int_{a}^{b} d r r & \left\{\left[\left|\frac{\partial}{\partial r} \delta \phi^{\ell}\right|^{2}+\frac{\ell^{2}}{r^{2}}\left|\delta \phi^{\ell}\right|^{2}\right]\left[1+\frac{\omega_{p b}^{2}(r)}{\omega_{c}^{2}-\omega_{p b}^{2}(r)}\right]\right. \\
& \left.-\frac{\ell\left|\delta \phi^{\ell}\right|^{2}}{r} \frac{\omega_{c} P}{\left[\omega_{r}-\ell \omega_{E}(r)\right]} \frac{\omega_{c}^{2}}{\left[\omega_{c}^{2}-\omega_{p b}^{2}(r)\right]^{2}} \frac{\partial}{\partial r} \omega_{p b}^{2}(r)\right\}
\end{aligned}
$$

and

$$
\begin{aligned}
\gamma=-\frac{D_{i}\left(\omega_{r}\right)}{\partial D_{r} / \partial \omega_{r}}=-\pi \ell & \int_{a}^{b} d r\left|\delta \phi^{\ell}\right|^{2} \frac{\omega_{c}^{3} \delta\left[\omega_{r}-\ell \omega_{E}(r)\right]}{\left[\omega_{c}^{2}-\omega_{p b}^{2}(r)\right]^{2}} \frac{\partial}{\partial r} \omega_{p b}^{2}(r) \\
& \times\left[\int_{a}^{b} d r\left|\delta \phi^{\ell}\right|^{2} \frac{\ell \omega_{c} P}{\left[\omega_{r}-\ell \omega_{E}(r)\right]^{2}} \frac{\omega_{c}^{2}}{\left[\omega_{c}^{2}-\omega_{p b}^{2}(r)\right]^{2}} \frac{\delta}{\partial r} \omega_{p b}^{2}(r)\right]^{-1}
\end{aligned}
$$

Equation (59) determines the real frequency $\omega_{r}$ (assuming that $\delta \phi^{\ell}$ is known), whereas the growth rate $\gamma$ is given by Eq. (60).

In circumstances where $\delta D_{r} / \delta w_{r}<0$, it follows from Eq. (60) that resonant instability exists whenever

$$
\left.\frac{\partial w_{p b}^{2}}{\partial r}\right|_{r=r_{0}}>0,
$$

where the resonant radius $r_{a}$ satisfies

$$
\omega_{r}-\ell \omega_{E}\left(r_{s}\right)=0
$$

Density profiles $n_{b}^{0}(r)$ with a gentle bump (Fig. 3) are prime candidates for such a weak resonant instability. A specific example is discussed in Sec. VI. 


\section{v. SUFFICIENT CONDITION FOR STABILITY IN THE \\ GUIDING-CENTER APPROXIMATION}

The stability analysis in Sec. IV.A represents a direct calculation of the sufficient condition [Eq. (55)] for the equilibrium density profile $n_{b}^{0}(r)$ to be stable for low-frequency flute perturbations. The calculation is direct in the sense that it makes explicit use of the eigenvalue equation (47). In this section, we give an indirect proof of this stability condition, based on global conservation constraints satisfied by the fully nonlinear macroscopic fluid equations in the guiding-center approximation.

In particular, in the present analysis, we adopt a cold-fluid guiding-center model in which electron inertial effects are neglected $(m \rightarrow 0)$ and the motion of a strongly magnetized electron fluid element is determined from

$$
E(x, t)+\frac{1}{c} V_{b}(x, t) \times B_{b} \hat{e}_{z}=0 .
$$

In the electrostatic approximation, $E=-\nabla \phi$ and Eq. (63) gives

$$
V_{b}(x, t)=-\frac{c}{B_{0}} \nabla \phi(x, t) \times \hat{e}_{z}
$$

for the perpendicular motion. In cylindrical geometry, Eq. (64) reduces to

$$
\begin{aligned}
& V_{r b}(r, \theta, t)=-\frac{c}{B_{0} r} \frac{\partial}{\partial \theta} \phi(r, \theta, t), \\
& V_{\theta b}(r, \theta, t)=\frac{c}{B_{0} r} \frac{\partial}{\partial r} \phi(r, \theta, t),
\end{aligned}
$$

where $\delta / \partial z=0$ has been assumed. The continuity equation, which relates the density $n_{b}(r, \theta, t)$ and flow velocity $V_{b}(r, \theta, t)$ is given by

$$
\frac{\partial}{\partial t} n_{b}+\frac{\partial}{\partial x} \cdot\left(n_{b} V_{b}\right)=0
$$

which reduces to

$$
\frac{\partial}{\partial t} n_{b}+V_{b} \cdot \frac{\partial}{\partial x} n_{b}=0,
$$

since $\nabla \cdot V_{b}=0$ for the electron flow in Eq. (64). Of course, Eqs. (64)-(66) must be supplemented by Poisson's equation 


$$
\nabla^{2} \phi(r, \theta, t)=4 \pi e n_{b}(r, \theta, t),
$$

which self-consistently relates the electrostatic potential $\phi(r, \theta, t)$ to the electron density $n_{b}(r, \theta, t)$.

Equations (64), (66) and (67) constitute a fully nonlinear description of the system evolution in the coldfluid guiding-center approximation with $m \rightarrow 0$ and $B_{0} \rightarrow \infty$. Expressing

$$
\begin{aligned}
n_{b}(r, \theta, t) & =n_{b}^{0}(r)+\delta n_{b}(r, \theta, t), \\
\phi(r, \theta, t) & =\phi_{0}(r)+\delta \phi(r, \theta, t),
\end{aligned}
$$

the boundary conditions enforced in solving Eqs. (64), (66) and (67) are

$$
\begin{gathered}
\phi_{0}(r=a)=0 \text { and } \phi_{0}(r=b)=V, \\
\left.\frac{\partial}{\partial r} \phi_{0}\right|_{r=a}=0
\end{gathered}
$$

and

$$
\frac{\partial}{\partial \theta} \delta \phi=0, \text { at } r=a \text { and } r=b .
$$

The equilibrium conditions in Eq. (69) correspond to space-charge limited flow with $E_{r}^{0}(r=a)=$ $-\partial \phi_{0} /\left.\partial r\right|_{r=a}=0$. Moreover, Eq. (70) assures that the tangential electric field and radial flow velocity are equal to zero at the cathode and at the anode, with

$$
\left.\begin{array}{c}
E_{\theta}=-\frac{1}{r} \frac{\partial}{\partial \theta} \delta \phi=0, \\
V_{r b}=-\frac{c}{B_{0} r} \frac{\partial}{\partial \theta} \delta \phi=0,
\end{array}\right\} \text { at } r=a \text { and at } r=b .
$$

Finally, because of periodicity in the $\theta$-direction,

$$
\int_{0}^{2 \pi} d \theta \frac{\partial}{\partial \theta} \psi=0,
$$

where $\psi$ represents any field or fluid variable or nonlinear combination thereof.

The macroscopic guiding center model based on Eqs. (64), (66) and (67) possesses certain global (spatially averaged) conservation constraints. Consider the quantity $\Delta U_{G}$ defined by 


$$
\Delta U_{G}=\int d^{2} x\left[G\left(n_{b}\right)-G\left(n_{b}^{0}\right)\right]
$$

where $G\left(n_{b}\right)$ is a smooth, differentiable function, and

$$
\int d^{2} x=\int_{0}^{2 \pi} d \theta \int_{a}^{b} d r r
$$

in cylindrical geometry. From Eq. (66) and $\nabla \cdot V_{b}=0$, it follows that

$$
\begin{aligned}
\frac{\partial}{\partial t} G\left(n_{b}\right) & =\frac{\partial G}{\partial n_{b}} \frac{\partial n_{b}}{\partial t}=-\frac{\partial G}{\partial n_{b}} V_{b} \cdot \nabla n_{b} \\
& =-V_{b} \cdot \nabla G\left(n_{b}\right)=-\nabla \cdot\left[G\left(n_{b}\right) V_{b}\right] .
\end{aligned}
$$

Therefore

$$
\begin{aligned}
\frac{d}{d t} \Delta U_{G} & =\int d^{2} x \frac{\partial}{\partial t} G\left(n_{b}\right) \\
& =-\int_{0}^{2 \pi} d \theta \int_{a}^{b} d r r\left[\frac{1}{r} \frac{\partial}{\partial r}\left(r V_{r b} G\right)+\frac{1}{r} \frac{\partial}{\partial \theta}\left(V_{\theta b} G\right)\right]=0 .
\end{aligned}
$$

The $\partial / \partial \theta$ contribution in Eq. (75) integrates to zero by virtue of periodicity in the $\theta$-direction [Eq. (72)]. The o/Or contribution in Eq. (75) integrates to zero because the radial flow velocity is equal to zero at the cathode $(r=a)$ and at the anode $(r=b)$ [Eq. (68)]. From Eq. (75), we conclude that

$$
\Delta U_{G}=\int d^{2} x\left[G\left(n_{b}\right)-G\left(n_{b}^{0}\right)\right]=\text { const. }
$$

A special case of Eg. (76) is the conservation of total charge

$$
\Delta U_{q}=-e \int d^{2} x\left(n_{b}-n_{b}^{0}\right)=\text { const. }=0,
$$

where the constant in Eq. (77) has been taken equal to zero. This is consistent for all time $t$ provided zero net charge is introduced into the system by the initial density perturbation, i.e., provided $\int d^{2} x \delta n_{b}(r, \theta, t=0)=$ o.

A further global conservation constraint is related to the density-weighted average radial location of guiding centers. Defining

$$
\Delta U_{r}=\int d^{2} x r^{2}\left(n_{b}-n_{b}^{0}\right)
$$


and making use of $\partial n_{b} / \partial t=-\nabla \cdot\left(n_{b} V_{b}\right)$ gives

$$
\begin{aligned}
\frac{d}{d t} \Delta U_{r} & =-\int_{0}^{2 \pi} d \theta \int_{a}^{b} d r r \cdot r^{2}\left[\frac{1}{r} \frac{\partial}{\partial r}\left(r n_{b} V_{r b}\right)+\frac{1}{r} \frac{\partial}{\partial \theta}\left(n_{b} V_{\theta b}\right)\right] \\
& =-\int_{0}^{2 \pi} d \theta \int_{a}^{b} d r\left[\frac{\partial}{\partial r}\left(r^{3} n_{b} V_{r b}\right)-2 r^{2} n_{b} V_{r b}\right]
\end{aligned}
$$

where the $\partial / \partial \theta$ contribution in Eq. (79) vanishes by virtue of periodicity in the $\theta$-direction [Eq. (72)]. Moreover, making use of $V_{r b}=0$ at $r=a$ and $r=b$ [Eq. (68)], the $(\theta / \partial r)\left(r^{3} n_{b} V_{r b}\right)$ term in Eq. (79) integrates to zero, which gives

$$
\frac{d}{d t} \Delta U_{r}=-\frac{2 c}{B_{0}} \int_{0}^{2 \pi} d \theta \int_{a}^{b} d r r n_{b} \frac{\theta}{B \theta} \phi .
$$

In Eq. (80), use has been made of $V_{r b}=-\left(c / r B_{0}\right) \partial \phi / \partial \theta$ [Eq. (65)] to eliminate the radial flow velocity $V_{r b}$ from the final term in Eq. (79). From $\nabla^{2} \phi=4 \pi e n_{b}$, Eq. (80) can be expressed as

$$
\begin{aligned}
\frac{d}{d t} \Delta U_{r} & =-\frac{c}{2 \pi e B_{0}} \int_{0}^{2 \pi} d \theta \int_{a}^{b} d r r\left[\frac{1}{r} \frac{\partial}{\partial r} r \frac{\partial \phi}{\partial r}+\frac{1}{r^{2}} \frac{\partial^{2} \phi}{\partial \theta^{2}}\right] \frac{\partial \phi}{\partial \theta} \\
& =-\frac{c}{2 \pi e B_{0}} \int_{0}^{2 \pi} d \theta \int_{a}^{b} d r\left[\frac{\partial}{\partial r}\left(r \frac{\partial \phi}{\partial r}\right)\right]\left[\frac{\partial \phi}{\partial \theta}\right]
\end{aligned}
$$

where the $\partial^{2} / \delta \theta^{2}$ contribution in Eq. (81) integrates to zero because of periodicity in the $\theta$-direction. Equation (81) can also be expressed exactly as

$$
\frac{d}{d t} \Delta U_{r}=-\frac{c}{2 \pi e B_{0}} \int_{0}^{2 \pi} d \theta \int_{a}^{b} d r\left[\frac{\partial}{\partial r}\left(r \frac{\partial \phi}{\partial r} \frac{\partial \phi}{\partial \theta}\right)-r \frac{\partial \phi}{\partial r} \frac{\partial}{\partial \theta} \frac{\partial \phi}{\partial r}\right] .
$$

The $\theta$-derivative term in Eq. (82) vanishes because of periodicity in the $\theta$-direction [Eq. (72)]. The term $(\theta / \partial r)[r(\partial \phi / \partial r)(\partial \phi / \partial \theta)]$ in Eq. (82) integrates to zero by virtue of $E_{\theta}=-(1 / r)(\partial \phi / \partial \theta)=0$ at the cathode $(r=a)$ and at the anode $(r=b)$ [Eq. (71)]. This gives

$$
\frac{d}{d t} \Delta U_{r}=0
$$

or equivalently,

$$
\Delta U_{r}=\int d^{2} x r^{2}\left(n_{b}-n_{b}^{0}\right)=\text { const. }
$$


A sufficient condition for stability follows directly from Eqs. (76) and (84). Defining an effective free energy function $\Delta F$ by

$$
\Delta F=\Delta U_{r}+\Delta U_{G}
$$

it follows that

$$
\Delta F=\int d^{2} x\left[r^{2}\left(n_{b}-n_{b}^{0}\right)+G\left(n_{b}\right)-G\left(n_{b}^{0}\right)\right]=\text { const. }
$$

is an exact (nonlinear) global constraint. Expressing $\delta n_{b}=n_{b}-n_{b}^{0}$, and Taylor expanding

$$
G\left(n_{b}\right)=G\left(n_{b}^{0}\right)+G^{\prime}\left(n_{b}^{0}\right)\left(\delta n_{b}\right)+\frac{1}{2} G^{\prime \prime}\left(n_{b}^{0}\right)\left(\delta n_{b}\right)^{2}+\cdots
$$

it follows that Eq. (86) can be expressed as

$$
\Delta F=\int d^{2} x\left\{\left[r^{2}+G^{\prime}\left(n_{b}^{0}\right)\right]\left(\delta n_{b}\right)+\frac{1}{2} G^{\prime \prime}\left(n_{b}^{0}\right)\left(\delta n_{b}\right)^{2}\right\}=\text { const. }
$$

correct to quadratic order in the density perturbation $\delta n_{b}=n_{b}(r, \theta, t)-n_{b}^{0}(r)$. The function $G\left(n_{b}^{0}\right)$ has been arbitrary up to this point. We now choose $G\left(n_{b}^{0}\right)$ to satisfy

$$
G^{\prime}\left(n_{b}^{0}\right)=-r^{2}
$$

so that $G^{\prime \prime}\left(n_{b}^{0}\right)=-\left(O n_{b}^{0} / \partial r\right)^{-1}$. Equation $(88)$ then becomes

$$
\Delta F=\frac{1}{2} \int d^{2} x \frac{1}{\left[-\delta n_{b}^{0} / \delta r^{2}\right]}\left(\delta n_{b}\right)^{2}=\text { const. }
$$

It follows trivially from Eq. (90) that for monotonic decreasing density profiles with

$$
\frac{1}{r} \frac{\partial n_{b}^{0}}{\partial r} \leq 0, \text { for } a \leq r \leq b
$$

the density perturbation $\delta n_{b}(r, \theta, t)$ cannot grow without bound, and the system is linearly stable. That is to say, Eq. (91) is a sufficient condition for stability in the context of the cold-fluid guiding-center model based on Eqs. (64), (66) and (67).

The analysis in this section illustrates the tremendous advantage of using global conservation constraints to determine a sufficient condition for stability. Nowhere has it been necessary to make direct use of a detailed normal-mode analysis or eigenvalue equation. 


\section{ANALYTIC SOLUTIONS TO ELECTROSTATIC EIGENVALUE EQUATION}

\section{A. Stable Surface Waves}

In this section, we make use of the approximate eigenvalue (47) to investigate stability properties for lowfrequency flute perturbations with $\left|\omega-\ell \omega_{E}\right|^{2} \ll \omega_{c}^{2}-\omega_{p}^{2}(r)$ and $\omega_{p}^{2}(r)<\omega_{c}^{2}$ [Eqs. (45) and (46)]. As a specific example that is analytically tractable, consider the rectangular density profile (Fig. 2) specified by

$$
n_{b}^{0}(r)= \begin{cases}\hat{n}_{b}=\text { const., } & a \leq r<r_{b} \\ & \\ 0, & r_{b}<r \leq b .\end{cases}
$$

From Eqs. (37) and (92), $\omega_{E}(r)=-c E_{r}^{0}(r) / r B_{0}$ can be expressed as (for $a \leq r<r_{b}$ )

$$
\omega_{E}(r)=\frac{\hat{\omega}_{p b}^{2}}{2 \omega_{c}}\left(\frac{r^{2}-a^{2}}{a^{2}}\right)
$$

where $\hat{\omega}_{p b}^{2}=4 \pi \hat{n}_{b} e^{2} / m=$ const. Because $\omega_{p b}^{2}(r)=\hat{\omega}_{p b}^{2}=$ const. for $a \leq r<r_{b}$ (Region I), the eigenvalue equation (47) reduces to

$$
\frac{1}{r} \frac{\partial}{\partial r} r \frac{\partial}{\partial r} \delta \phi_{I}^{\ell}-\frac{\ell^{2}}{r^{2}} \delta \phi_{I}^{\ell}=0, \quad a \leq r<r_{b}
$$

within the electron annulus. Moreover, because $\omega_{p b}^{2}(r)=0$ for $r_{b}<r \leq b$ (Region II), the eigenvalue equation (47) can be expressed as

$$
\frac{1}{r} \frac{\partial}{\partial r} r \frac{\partial}{\partial r} \delta \phi_{I I}^{\ell}-\frac{\ell^{2}}{r^{2}} \delta \phi_{I I}^{\ell}=0, \quad r_{b}<r \leq b,
$$

in the vacuum region between the anode and surface of the electron annulus. The solutions to Eqs. (94) and (95) that satisfy $\delta \phi_{I}^{\ell}(r=a)=0=\delta \phi_{I I}^{\ell}(r=b)$ and are continuous at $r=r_{b}$ are given by

$$
\delta \phi_{1}^{l}(r)=A\left[\left(\frac{r}{a}\right)^{\ell}-\left(\frac{a}{r}\right)^{\ell}\right], \quad a \leq r<r_{b}
$$

and

$$
\delta \phi_{I I}^{\ell}(r)=A\left[\left(\frac{r}{b}\right)^{\ell}-\left(\frac{b}{r}\right)^{l}\right]\left[\left(\frac{r_{b}}{a}\right)^{\ell}-\left(\frac{a}{r_{b}}\right)^{l}\right] \times\left[\left(\frac{r_{b}}{b}\right)^{\ell}-\left(\frac{b}{r_{b}}\right)^{l}\right]^{-1}, r_{b}<r \leq b .
$$


The remaining boundary condition on $(\theta / \partial r) \delta \phi^{\ell}$ at $r=r_{b}$ is obtained by integrating the eigenvalue equation (47) across the surface of the plasma at $r=r_{b}$. Multiplying Eq. (47) by $r$ and integrating from $r_{b}(1-\epsilon)$ to $r_{b}(1+\epsilon)$, with $\epsilon \rightarrow 0_{+}$, gives

$$
\begin{gathered}
{\left[r \frac{\delta}{\delta r} \delta \phi_{I I}^{\ell}\right]_{r=n}-\left[1+\frac{\hat{\omega}_{p b}^{2}}{\left(\omega_{c}^{2}-\hat{\omega}_{p b}^{2}\right)}\right]\left[r \frac{\delta}{\delta r} \delta \phi_{I}^{\ell}\right]_{r=r}} \\
=\frac{\ell \delta \phi^{\ell}\left(r=r_{b}\right) \omega_{c}}{\left[\omega-\ell \omega_{E}\left(r_{b}\right)\right]} \frac{\hat{\omega}_{p b}^{2}}{\left(\omega_{c}^{2}-\hat{\omega}_{p b}^{2}\right)}
\end{gathered}
$$

where $\hat{\omega}_{p b}^{2}=4 \pi \hat{n}_{b} e^{2} / m$, and

$$
\omega_{E}\left(r_{b}\right)=\frac{\vec{\omega}_{p b}^{2}}{2 \omega_{c}}\left(\frac{r_{b}^{2}-a^{2}}{a^{2}}\right) .
$$

Equation (98) relates the discontinuity in perturbed radial electric field to the perturbed surface charge density at $r=r_{b}$. It is useful to define

$$
\begin{aligned}
& \hat{\epsilon}_{\perp}=1+\frac{\hat{\omega}_{p b}^{2}}{\left(\omega_{c}^{2}-\hat{\omega}_{p b}^{2}\right)}=\frac{\omega_{c}^{2}}{\left(\omega_{c}^{2}-\hat{\omega}_{p b}^{2}\right)^{\prime}} \\
& \hat{\Omega}_{D}=\frac{\hat{\omega}_{p b}^{2}}{2 \omega_{c}}
\end{aligned}
$$

and

$$
\frac{1}{g_{l}}=\left[\left(\frac{r_{b}}{b}\right)^{l}+\left(\frac{b}{r_{b}}\right)^{l}\right] \times\left[\left(\frac{r_{b}}{b}\right)^{\ell}-\left(\frac{b}{r_{b}}\right)^{l}\right]^{-1}-\bar{\epsilon}_{\perp}\left[\left(\frac{r_{b}}{a}\right)^{l}+\left(\frac{a}{r_{b}}\right)^{l}\right] \times\left[\left(\frac{r_{b}}{a}\right)^{l}-\left(\frac{a}{r_{b}}\right)^{l}\right]^{-1},
$$

where $g_{l}$ is an effective geometric factor. Substituting Eqs. (96) and (97) into Eq. (98) then gives

$$
\frac{1}{g_{\ell}}=\frac{2 \hat{\Omega}_{D_{\perp}^{2}}}{\left[\omega-\ell \omega_{E}\left(r_{b}\right)\right]^{\prime}}
$$

or equivalently,

$$
\omega-\ell \omega_{E}\left(r_{b}\right)=\left(2 g^{\hat{\epsilon}} \perp\right) \hat{\Omega}_{D}
$$

Note that Eq. (98) has played the role of an effective dispersion relation that determines the eigenfrequency $\omega$. From Fq. (103), for the rectangular density profile in Eq. (92) and Fig. 2, the system supports only stable oscillations $(\operatorname{Imw}=0)$. On the other hand, a completely analogous analysis of the eigenvalue equation (47) can be 
carried out for the hollow density profile illustrated in Fig. 4, where the inner surface of the electron annulus is separated from the cathode. In this case, there are charge perturbations on the inner surface $\left(r=r_{b}^{-}\right)$as well as the outer surface $\left(r=r_{b}^{+}\right)$of the annulus, and the interaction leads to the familiar diocotron instability modified to include plasma dielectric effects with $\hat{\epsilon}_{\perp}-1>0$.

To conclude this section, we simplify the expression for the geometric factor $g$ in Eq. (101) for a moderate-aspect-ratio diode with

$$
a \gg d=(b-a)
$$

and for (low) harmonic numbers $\ell$ satisfying

$$
\frac{\ell d}{a} \ll 1
$$

To leading order, Eq. (101) gives the approximate expression for $q$

$$
\frac{1}{g_{\ell}}=\left[1-\frac{a+d}{\ell\left[d-\left(r_{b}-a\right)\right]}\right]-\hat{\epsilon}_{\perp}\left[1+\frac{a}{\ell\left(r_{b}-a\right)}\right]
$$

where $\ell\left(r_{b}-a\right) / a \ll 1$ is assumed, and $d=b-a$ is the anode-cathode spacing. We denote (Fig. 5) the thickness of the annulus by $\Delta_{a}$ and the width of the vacuum region by $\Delta_{v}$, where

$$
\begin{aligned}
& \Delta_{a}=r_{b}-a \\
& \Delta_{v}=d-\left(r_{b}-a\right)=b-r_{b}
\end{aligned}
$$

For $\ell \Delta_{a} / a \ll 1$ and $\ell \Delta_{v} / a \ll 1$, Eq. (106) can be approximated by

$$
g e=-\frac{\ell \Delta_{v} / b}{1+\hat{\epsilon}_{\perp}\left(\Delta_{v} a / \Delta_{a} b\right)}
$$

For a thin annulus, it follows from Eq. (93) that $\omega_{E}(r)$ can be approximated by

$$
\omega_{E}(r)=2 \hat{\Omega}_{D}\left(\frac{r-a}{a}\right)
$$

and $\omega_{E}\left(r_{b}\right)$ by

$$
\dot{\omega}_{E}\left(r_{b}\right)=2 \frac{\Delta_{a}}{a} \hat{\Omega}_{D}
$$

where $\hat{\Omega}_{D}=\hat{\omega}_{p b}^{2} / 2 \omega_{c}$. For $b \simeq a$, we substitute the approximate expression (108) for $g_{\ell}$ into Eq. (103). This gives 


$$
\omega-\ell \omega_{E}\left(r_{b}\right)=-\frac{\hat{\epsilon}_{\perp}}{1+\hat{\epsilon}_{\perp} \Delta_{v} / \Delta_{a}} 2 \ell \frac{\Delta_{v}}{a} \hat{\Omega}_{D}
$$

where $\omega_{E}\left(r_{b}\right)$ is defined in Eq. (110). Since $\ell d / a \ll 1$ has been assumed in Eq. (111), it follows that the lowfrequency assumption, $\left|\omega-\ell \omega_{E}\right|^{2} \ll \omega_{c}^{2}-\hat{\omega}_{p}^{2}$ in Eq. (46), is readily satisfied for moderate values of $\hat{\omega}_{p b}^{2} / \omega_{c}^{2}$.

\section{B. Resonant Diocotron Instability}

We now consider circumstances where the main density region $\left(a \leq r<r_{b}\right)$ is seeded with a low-density component of circulating electrons (Fig. 6). In the region $a \leq r<r_{b}, \omega_{E}(r)=-c E_{r}^{0}(r) / B_{0}$ is still given approximately by Eq. (109) because $E_{r}^{0}(r)$ is determined primarily by the main density component $\left(\hat{n}_{b}\right)$ in Fig. 6. That is, $\omega_{E}(r)$ can be approximated by

$$
\omega_{E}(r)=2 \hat{\Omega}_{D}\left(\frac{r-a}{a}\right), \quad a \leq r<r_{b}
$$

in the region of the low-density bump in Fig. 6. Moreover, the analytic results in Sec. VI.A represent excellent approximations for the eigenfunctions in Regions I and II [Eqs. (96) and (97)], and for the real oscillation frequency $\omega_{r}=$ Rew [Eq. (111)] in circumstances where the bump density is much less than $\hat{n}_{b}$. That is, $\omega_{r}=$ Rew is given approximately by

$$
\omega_{r}-\ell \omega_{E}\left(r_{b}\right)=-\frac{\epsilon_{\perp}}{1+\hat{\epsilon}_{\perp} \Delta_{v} / \Delta_{a}} 2 \ell \frac{\Delta_{v}}{a} \hat{\Omega}_{D}
$$

for a large-aspect-ratio diode with $\ell d / a \ll 1$.

In Sec. IV.B, we derived a formal expression [Eq. (60)] for the growth rate $\gamma=I m \omega$ assuming (weak) resonant instability driven by a gentle bump in the density profile $n_{b}^{0}(r)$. From Eqs. (61) and (62), the condition for instability is

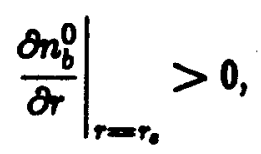

where $r_{s}$ solves the resonance condition

$$
\omega_{r}-\ell \omega_{E}\left(r_{0}\right)=0
$$

Substituting Eqs. (112) and (113) into Eq. (115), and solving for $r_{0}$ gives 


$$
r_{0}=r_{b}\left(1-\frac{\hat{c}_{\perp}}{1+\hat{\epsilon}_{\perp} \Delta_{v} / \Delta_{a}} \frac{\Delta_{v}}{r_{b}}\right)
$$

which determines the resonant radius $r_{\mathrm{a}}$ that satisfies Eq. (115). For example, if $\dot{\epsilon}_{\perp}=1.5$ and $\Delta_{v} / \Delta_{a}=2 / 3$, then $r_{b}-r_{b}=-0.5 \Delta_{a}$ follows from Eq. (116).

The expression for growth rate $\gamma$ is given in Eq. (60). Assuming that $r_{b}$ satisfies $a<r_{0}<r_{b}$ [Eq. (116) and Fig. 6], then $D_{i}\left(\omega_{r}\right)=\operatorname{ImD}(\omega)$ is given by [Eq. (60)]

$$
D_{i}\left(\omega_{r}\right)=\left.\frac{\pi a \hat{\varepsilon}_{\perp}^{2}}{\left(2 \omega_{c} \Omega_{D}\right)}\left|\delta \phi_{I}^{l}\right|_{r=r,}^{2} \frac{\delta}{\delta r} \omega_{p b}^{2}(r)\right|_{r=r_{c}},
$$

where use has been made of $\partial \omega_{E} /\left.\partial r\right|_{r=r_{s}}=2 \hat{\Omega}_{d} / a$ [Eq. (12)]. In Eq. (117), $\delta \phi_{J}^{\ell}(r)$ is approximated by Eq. (96), i.e., by the eigenfunction in Region I in the absence of density bump. Similarly, $\partial D_{r} / \partial w_{r}$ is given by [Eq. $(60)]$

$$
\frac{\partial D_{r}}{\partial \omega_{r}}=-\left|\delta \phi_{I}^{\ell}\right|_{r=r_{b}}^{2} \frac{2 \ell \Omega_{D_{\perp}^{\prime}}}{\left[\omega_{r}-\ell \omega_{E}\left(r_{b}\right)\right]^{2}}
$$

where $\delta \phi_{I}^{\ell}(r)$ is approximated by $(96), \hat{\epsilon}_{\perp}$ is defined in Eq. (100), and $\hat{\Omega}_{D}=\hat{\omega}_{p b}^{2} / 2 \omega_{c}$. Note from Eq. (60), Eq. (118) and Fig. 6 that the main contribution to $\partial D_{r} / \partial w_{r}$ comes from the density discontinuity at the surface $\left(r=r_{b}\right)$ of the electron annulus. Combining Eqs. (117) and (118), the growth rate $\gamma=-D_{i} /\left(\partial D_{r} / \partial w_{r}\right)$ is

$$
\gamma=\left.\frac{\pi \hat{\epsilon}_{\perp}}{4 \ell} \frac{\left.\mid \omega_{r}-\ell \omega_{E}\left(r_{b}\right)\right]^{2}}{\hat{\Omega}_{D}^{2}} \frac{\left|\delta \phi_{I}^{\ell}\right|_{\text {ras }}^{2}}{\left|\delta \phi_{I}^{\ell}\right|_{r=r_{b}}^{2}} \frac{a}{\omega_{c}} \frac{\partial}{\partial r} \omega_{p b}^{2}\right|_{r=r_{c}},
$$

where $\omega_{r}-\ell \omega_{E}\left(r_{b}\right)$ is given in Eq. (113). From Eqs. (96) and (97),

$$
\frac{\left|\delta \phi_{I}^{l}\right|_{r=r_{b}}^{2}}{\left|\delta \phi_{I}^{l}\right|_{r=r_{b}}^{2}}=\left[\left(\frac{r_{b}}{a}\right)^{l}-\left(\frac{a}{r_{b}}\right)^{l}\right]^{2} \times\left[\left(\frac{r_{b}}{a}\right)^{l}-\left(\frac{a}{r_{b}}\right)^{l}\right]^{-2} \simeq\left(\frac{r_{b}-a}{r_{b}-a}\right)^{2},
$$

for a large-aspect-ratio diode with $\ell\left(r_{b}-a\right), \ell\left(r_{b}-a\right) \ll a$. Defining $r_{b}-a=\Delta_{a}$ (Fig. 6), and making use of $r_{b}-a=r_{b}-a-\left(r_{b}-r_{b}\right)=\Delta_{a}\left(1+\dot{\epsilon}_{\perp} \Delta_{v} / \Delta_{a}\right)^{-1}$ [Eq. (116)], the ratio in Eq. (120) can be approximated by

$$
\frac{\left|\delta \phi_{I}^{\ell}\right|_{r=r_{e}}^{2}}{\left|\delta \phi_{Y}\right|_{r=n}^{2}}=\frac{1}{\left(1+\epsilon_{\perp} \Delta_{v} / \Delta_{a}\right)^{2}}
$$

Returning to Eq. (119), we normalize the growth rate $\gamma$ to $\ell \omega_{E}\left(r_{b}\right)=2 \ell \grave{\Omega}_{D} \Delta_{a} / a$, where $\Delta_{a}=r_{b}-a$ and $\omega_{E}\left(r_{b}\right)$ is given in Eq. (110). Making use of Eqs. (111) and (121), the normalized growth rate $\gamma / \ell \omega_{E}\left(r_{b}\right)$ in Eq. (119) can be expressed as 


$$
\frac{\gamma}{\ell \omega_{E}\left(r_{b}\right)}=\left.\frac{\pi \hat{\epsilon}_{\perp}^{3} \Delta_{a}^{2} \Delta_{v}^{2}}{\left(\Delta_{a}+\hat{\epsilon}_{\perp} \Delta_{v}\right)^{4}} \frac{\Delta_{a}}{\dot{\omega}_{p b}^{2}} \frac{\partial}{\partial r} \omega_{p b}^{2}(r)\right|_{r=r_{b}},
$$

where $\hat{\omega}_{p b}^{2}=2 \omega_{c} \hat{\Omega}_{D}=4 \pi \hat{n}_{b} e^{2} / m$ is the plasma frequency-squared of the main density component $\left(\hat{n}_{b}\right)$ in Fig. 6.

Measured in units of $\ell \omega_{E}\left(r_{b}\right)$, the growth rate $\gamma$ in Eq. (122) can be substantial. The resonant diocotron instability discussed in this section may well be one of the most important instabilities characteristic of electron flow in diodes. Although monotonic decreasing profiles with $\delta n_{b}^{0} / \partial r \leq 0$ are stable [Figs. 3(a) or 5], the introduction of a low-density circulating electron component [Figs. 3(b), 3(c) or 6] can lead to resonant instability. 


\section{NUMERICAL SOLUTION TO ELECTROSTATIC EIGENVALUE EQUATION}

In this section, we make use of the exact electrostatic eigenvalue equation (34) to investigate numerically the stability properties of a variety of equilibrium profiles $n_{b}^{0}(r)$.

\section{A. Eigenvalue Equation}

For nonrelativistic, cold-fluid flow, the exact electrostatic eigenvalue equation in cylindrical geometry is given by Eq. (34). It is convenient to introduce the dimensionless radial coordinate

$$
R=\frac{r}{a}
$$

where $r=a$ is the location of the cathode (Fig. 1). Moreover, all frequencies are normalized to $\omega_{c}=e B_{0} / m c$ with

$$
\begin{aligned}
\dot{\omega} & \equiv \frac{\omega}{\omega_{c}}, \quad \hat{\omega}_{E}(r) \equiv \frac{\omega_{E}(r)}{\omega_{c}}=\frac{1}{r^{2} \omega_{c}^{2}} \int_{a}^{r} d r^{\prime} r^{\prime} \omega_{p b}^{2}(r), \\
\hat{\omega}_{p b}^{2}(r) & \equiv \frac{\omega_{p b}^{2}(r)}{\omega_{c}^{2}}=\frac{4 \pi n_{b}^{0}(r) e^{2}}{m \omega_{c}^{2}}, \\
\hat{\omega}_{b}^{-}(r) & \equiv \frac{\omega_{b}^{-}(r)}{\omega_{c}}=\frac{1}{2}\left\{1-\left[1-4 \hat{\omega}_{E}(r)\right]^{1 / 2}\right\} .
\end{aligned}
$$

Making use of Eqs. (123) and (124), the eigenvalue equation (34) can be expressed in the equivalent form

$$
\begin{aligned}
\frac{1}{R} \frac{\partial}{\partial R} & \left\{R\left[1-\frac{\hat{\omega}_{p b}^{2}}{\left(\hat{\omega}-\ell \hat{\omega}_{b}^{-}\right)^{2}-\left(1-\hat{\omega}_{p b}^{2}-2 \hat{\omega}_{E}\right)}\right] \frac{\partial}{\partial R} \delta \phi^{\ell}\right\} \\
& -\frac{\ell^{2}}{R^{2}}\left[1-\frac{\hat{\omega}_{p b}^{2}}{\left(\hat{\omega}-\ell \hat{\omega}_{b}^{-}\right)^{2}-\left(1-\hat{\omega}_{p b}^{2}-2 \omega_{E}\right)}\right] \delta \phi^{\ell} \\
& =\frac{\ell \delta \phi^{\ell}}{R} \frac{1}{\left(\hat{\omega}-\ell \hat{\omega}_{b}^{-}\right)} \frac{\partial}{\partial R}\left[\frac{\hat{\omega}_{p b}^{2}\left(1-4 \hat{\omega}_{E}\right)^{1 / 2}}{\left(\hat{\omega}-\ell \hat{\omega}_{b}^{-}\right)^{2}-\left(1-\dot{\omega}_{p b}^{2}-2 \hat{\omega}_{E}\right)}\right],
\end{aligned}
$$

where $k_{z}=0$ is assumed. and the dimensionless frequencies $\hat{\omega}, \hat{\omega}_{p b}^{2}(r)$, and $\hat{\omega}_{E}(r)$ are defined in Eq. (124). The equilibrium boundary condition assumed in Eq. (125) is $E_{r}^{0}(r=a)=0$ at the cathode, which corresponds to $\dot{\omega}_{E}(r=a)=0$. Note also that the eigenvalue equation (125) is exact within the context of the present 
electrostatic model based on the nonrelativistic cold-fluid-Poisson equations. That is, unlike the approximate eigenvalue cquations analyzed in Secs. IV-VI, there is no a priori assumption in Eq. (125) that $\omega_{E}(r) \ll \omega_{c}$ [as in Eq. (39)], that the perturbation frequency is low [as in Eq. (46)], or that the diode aspect ratio is large [as in Eq. (104)].

In Secs. VII.B and VII.C, Eq. (125) is solved numerically for the real oscillation frequency $\omega_{r}=$ Rew, the growth rate $\gamma=I \mathrm{mw}$, and the eigenfunction $\delta \phi^{\ell}(r)$ subject to the boundary conditions

$$
\delta \phi^{\ell}=0, \text { at } r=a \text { and at } r=b
$$

Depending on the choice of equilibrium density profile $n_{b}^{0}(r)$, the solutions to $\mathrm{Eq}$. (125) correspond to wcakly unstable oscillations (Sec. VII.B) or to strong instability (Sec. VII.C).

\section{B. Weakly Unstable Oscillations}

As a first example of an equilibrium density profile that gives weakly unstable oscillations, consider the rectangular density profile specified by (Fig. 2)

$$
n_{b}^{0}(r)= \begin{cases}\hat{n}_{b}=\text { const., } & a \leq r<r_{b} \\ 0, & r_{b}<r \leq b .\end{cases}
$$

Equation (125) has been solved numerically for the complex eigenfrequency $\omega=\omega_{r}+i \gamma$ and eigenfunction $\delta \phi^{\ell}(r)$ assuming $r_{b} / a=2$ and $b / a=3$. Typical results are illustrated in Fig. 7 , where $\omega_{r}=$ Rew and $\gamma=\operatorname{Im} \omega$ are tabulated versus azimuthal mode number $\ell=1,2,3,4,5$, for the low-frequency branch that solves Eq. (125). The values chosen for the dimensionless self-field parameter $s \equiv 4 \pi \hat{n}_{b} m c^{2} / B_{0}^{2}=\omega_{p b}^{2} / \omega_{c}^{2}$ correspond to $s=0.5$ [Fig. 7(a)] and $s=0.2$ [Fig. 7(b)]. Note from Figs. 7(a) and 7(b) that the system is weakly unstable $\left(\gamma \ll\left|\omega_{r}\right|\right)$ for the choice of rectangular density profile in Eq. (127). Moreover, the collisionless growth is weakest for low values of electron density (small values of $s$ ).

As a second example, we consider the bell-shaped density profile specified by (Fig. 8) 


$$
n_{b}^{0}(r)= \begin{cases}\hat{n}_{b}\left[1-\frac{(r-a)^{2}}{\left(r_{b}-a\right)^{2}}\right]^{2}, & a \leq r<r_{b}, \\ 0, & n_{b}<r \leq b .\end{cases}
$$

Note from Eq. (128) that $n_{b}^{0}(r)$ decreases monotonically from $n_{b}$ at $r=a$ to zero at $r=r_{b}$. The complex eigenfrequency $\omega=\omega_{r}+i \gamma$ and eigenfunction $\delta \phi^{\ell}(r)$ has been determined numerically from Eq. (125) for the choice of density profile in Eq. (128). Typical results are illustrated in Fig. 9 where $\omega_{r}$ and $\gamma$ are tabulated versus $s=4 \pi \hat{n}_{b} m c^{2} / B_{0}^{2}=\omega_{p b}^{2}(r=a) / \omega_{c}^{2}$ for mode number $\ell=2$, and $s$ in the range $0.48 \leq 8 \leq 1$. For $8 \leq 0.45$, it is found that the system is stable $(\gamma=\operatorname{Imw} \leq 0)$. For $8=0.48$, the onset of instability occurs for $\ell=2$ (Fig. 9). On the other hand, as $s$ is increased to $s=1$ it is found that the instability bandwidth increases to include $\ell=2,3,4$, and that maximum growth for $s=1$ occurs for $\ell=2$, where $\operatorname{Im} w=0.0977$ and $R e w / \omega_{c}=0.248$.

It is useful to define a resonant radius $r_{0}$ by the resonance condition

$$
\omega_{r}-\ell \omega_{E}\left(r_{0}\right)=0
$$

where $\omega_{r}=$ Rew solves Eg. (125). It is readily shown for the two numerical examples analyzed in this section that $r_{s}$ is located in the region where the electron density is non-zero (Figs. 10 and 11), i.e.,

$$
a<r_{0}<r_{\text {. }}
$$

Unlike the simplified approximate eigenvalue equations analyzed in Secs. IV and VI, it is important to recognize that the complete eigenvalue equation (125) provides collisionless dissipation even when $\delta w_{p b}^{2} /\left.\theta_{r}\right|_{r-r_{b}}=$ 0. This is evident from Fig. 7, where the modes are weakly growing for the choice of rectangular density profile in Eq. (127) (where $\delta \omega_{p b}^{2} /\left.\partial r\right|_{r=r_{0}}=0$ is trivially satisfied). The reason for this (negative) dissipation is readily traced to driving terms proportional to $\partial \omega_{b}^{-} / \theta r \neq 0$ on the right-hand side of Eq. (125). It is precisely such terms that are neglected in the approximate eigenvalue equation (47) either by virtuc of the assumptions $\omega_{E}(r) \ll \omega_{c}\left[\right.$ [Eq. (39)] or $\left|\omega-\ell \omega_{E}(r)\right|^{2} \ll \omega_{c}^{2}-\omega_{p}^{2}(r)[$ Eq. (46)] used in obtaining Eq. (47).

'To conclude this section, numerical plots of $n_{b}^{0}(r), \omega_{b}^{-}(r), \omega_{r}-\ell \omega_{b}^{-}(r), \operatorname{Re} \phi^{\prime}(r)$ and $I m \delta \phi^{\ell}(r)$ versus $r$ are presented in Figs. 10 and 11 for the two cases analyzed in Figs. 7 and 9, respectively. The parameters in Fig. 
10 correspond to $s=0.5$ and $\ell=4$ (rectangular density profile) and in Fig. 11 to $s=1.0$ and $\ell=2$ (bellshaped density profile).

\section{Strong Diocotron Instability in a Hollow Electron Beam}

As an example in which Eq. (125) predicts strong diocotron instability, we now consider the hollow electron density profile specified by (Fig. 4)

$$
n_{b}^{0}(r)=\left\{\begin{array}{cl}
0, & a \leq r<r_{b}^{-}, \\
\hat{n}_{b}=\text { const., } & r_{b}^{-}<r<r_{b}^{+}, \\
0, & r_{b}^{+}<r<b,
\end{array}\right.
$$

where the electron density $\left(\hat{n}_{b}\right)$ is constant in the beam interior. Heretofore, the diocotron instability corresponding to the choice of density profile in Eq. (131) has been analyzed only for the low-density case $\left(s=\omega_{p b}^{2} / \omega_{c}^{2}=4 \pi \hat{n}_{b} m c^{2} / B_{0}^{2} \ll 1\right)$ using a highly simplified (and approximate) form of the eigenvalue equation (125). In the present analysis, the complete eigenvalue equation (125) is solved numerically assuming $r_{b}^{-} / a=7 / 5, r_{b}^{+} / a=2$, and $b / a=3$. Typical results are illustrated in Fig. 12, where $\omega_{r}=$ Rew and $\gamma=\operatorname{Imw}$ are tabulated versus azimuthal mode number $\ell$ for $s=0.5$ [Fig. 12(a)] and $s=0.2$ [Fig. 12(b)] for the low-frequency branch that solves Eq. (125). Note from Fig. 12 that the instability growth rate $\gamma=I m \omega$ is strongest at high density. Moreover, the growth rate in Fig. 12 (hollow density profile) are substantially larger than the growth rates in Fig. 7 (rectangular density profile in contact with the cathode). It should also be pointed out that the growth rate is reduced if the outer or inner conductors are brought closer to the surface of the electron plasma (i.e., smaller $r_{b}^{-} / a$ or larger $\left.r_{b}^{+} / b\right)$. Finally, numerical plots of $n_{b}^{0}(r), \omega_{b}^{-}(r), \omega_{r}-\ell \omega_{b}^{-}(r)$, $\operatorname{Re} \delta \phi^{\ell}(r)$ and $I m \delta \phi^{\ell}(r)$ versus $r$ are shown in Fig. 13 for the case corresponding to $\ell=2$ and $s=0.5$ in Fig. 12 .

To conclude Sec. VII, we have also solved the exact eigenvalue equation (125) numerically for the highfrequency branch. For all of the profiles and parameter ranges considered earlier in Sec. VII. it is found that the high-frequency branch is stable $(\gamma=I m \omega \leq 0)$. 


\section{CONCLUSIONS}

In the present analysis, we have made use of a macroscopic, cold-fluid model (Secs. II and III) to investigate electrostatic stability properties of nonrelativistic sheared electron flow in a cylindrical diode with strong applied axial magnetic field $B_{0} \hat{e}_{\boldsymbol{z}}$. After reviewing the cold-fluid equilibrium properties, the linearized fluid-Poisson equations (25)-(27) are used in Sec. III to investigate stability behavior for electrostatic perturbations about a nonneutral cylindrical equilibrium characterized by (general) electron density profile $n_{b}^{0}(r)$ and self-consistent azimuthal velocity profile defined by $V_{\theta b}^{0}(r)=\omega_{b}^{-}(r) r$ [Eq. (15)]. For perturbations with complex oscillation frequency $\omega=\omega_{r}+i \gamma$, axial wavenumber $k_{z}$, and azimuthal harmonic number $\ell$, the linearized fluid-Poisson equations (29)-(33) were combined to give the eigenvalue cquation [Eq. (34)]

$$
\begin{gathered}
\frac{1}{r} \frac{\partial}{\partial r}\left\{r\left[1-\frac{\omega_{p b}^{2}}{\nu_{b}^{2}}\right] \frac{\partial}{\partial r} \delta \phi^{\ell}\right\}-\frac{\ell^{2}}{r^{2}}\left[1-\frac{\omega_{p b}^{2}}{\nu_{b}^{2}}\right] \delta \phi^{\ell}-k_{z}^{2}\left[1-\frac{\omega_{p b}^{2}}{\left(\omega-\ell \omega_{b}^{-}\right)^{2}}\right] \delta \phi^{\ell} \\
=-\frac{\ell \delta \phi^{\ell}}{r} \frac{1}{\left(\omega-\ell \omega_{b}^{-}\right)} \frac{\partial}{\partial r}\left[\frac{\omega_{p b}^{2}}{\nu_{b}^{2}}\left(2 \omega_{b}^{-}-\omega_{c}\right)\right] .
\end{gathered}
$$

The eigenvalue equation (34) provides an exact cold-fluid description of electrostatic stability properties, assuming nonrelativistic electron flow. For a moderate-aspect-ratio diode with $d \ll a$, the eigenvalue equation (34) was further simplified to give the approximate result in Eq. (43). In Secs. IV and VI, we analyzed Eq. (43) for the special case of low-frequency flute perturbations with

$$
k_{z}=0 \text { and }\left|\omega-\ell \omega_{E}(r)\right|^{2} \ll \omega_{c}^{2}-\omega_{p b}^{2}(r),
$$

assuming that the electron density is below the condition for Brillouin flow, i.e., $\omega_{p b}^{2}(r)<\omega_{c}^{2}$. In this case, the eigenvalue equation (43) can be approximated by Eq. (47). In Sec. IV.A, we made direct use of the eigenvalue equation (47) to show that

$$
\frac{\theta}{\delta r} \omega_{p b}^{2}(r) \leq 0
$$

over the interval $a \leq r \leq b$ is a sufficient condition to assure electrostatic stability. That is, equilibrium density profiles that decrease monotonically from the cathode to the anode are electrostatically stable. For the special case of weak resonant diocotron instability with growth rate $\gamma=I m \omega \ll\left|\omega_{r}\right|$, a formal expression for the growth rate $\gamma$ was derived in Sec. IV.B [Eq. (60)]. In Sec. VI, analytic solutions to the electrostatic eigenvalue equation (47) were determined both for stable surface modes on an annular electron beam (Sec. VI.A), and for weak resonant diocotron instability driven by a small density bump with $\delta \omega_{p b}^{2} /\left.O r\right|_{r=r_{e}}>0$ (Sec. VI.B). 
Because of the very general nature of the stability theorem obtained directly from the eigenvalue equation (47), we also developed an indirect proof that $\partial n_{b}^{0} / \partial r \leq 0$ is a sufficient condition for stability (Sec. V). The analysis in Sec. $V$ was based on a cold-fluid guiding-center model in which electron inertial effects are neglected $\left(m \rightarrow 0\right.$ and $\left.B_{0} \rightarrow \infty\right)$ and the motion of an electron fluid elcment is determined from $V_{b}=$ $\left(-c / B_{0}\right) \nabla \phi \times \hat{e}_{z}$. Making use of the continuity equation for $n_{b}(r, \theta, t)$ and Poisson's equation for $\phi(r, \theta, t)$, it was shown that $\Delta U_{G}=\int d^{2} r\left[G\left(n_{b}\right)-G\left(n_{b}^{0}\right)\right]=$ const. [Eq. (76)] and $\Delta U_{r}=\int d^{2} r r^{2}\left(n_{b}-n_{b}^{0}\right)=$ const. [Eq. (84)] are exact global conservation constraints. Note that Eq. (84) corresponds to conservation of densityweighted average radial guiding center location. Defining the effective free energy $\Delta F=\Delta U_{r}+\Delta U_{G}$, it was shown in Sec. V that $\delta n_{b}^{0} / \partial r \leq 0$ over the interval $a \leq r \leq b$ is a sufficient condition for electrostatic stability [Eq. (91)]. We reiterate that the indirect proof of this very powerful stability theorem in Sec. $\mathrm{V}$ has been based on a cold-fluid guiding-center model with $m \rightarrow 0$ and $B_{0} \rightarrow \infty$.

Finally, to conclude this paper, in Sec. VII we solved numerically the exact electrostatic eigenvalue equation (34) for a wide range of electron density profiles $n_{b}^{0}(r)$ leading to weak and strong instability driven by velocity shear.

\section{ACKNOWLEDGMENTS}

This work was supported by Sandia National Laboratories and in part by the Office of Naval Research. It is a pleasure to acknowledge the benefit of useful discussions with Richard Aamodt. 


\section{REFERENCES}

1. D.J. Johnson, G.W. Kuswa, A.V. Farnsworth Jr., J.P. Quintenz, R.J. Leeper, E.J.T, Burns and S. Humphries Jr., Phys. Rev. Lett. 42, 610 (1979).

2. J.P. Vandevender, J.P. Quintenz, R.J. Leeper, D.J. Johnson and J.T. Crow, J. Appl. Phys. 52 (1981).

3. T.C. Genoni, M.R. Franz, R.B. Miller and J.W. Poukey, J. Appl. Phys. 52, 2646 (1981).

4. D.J. Johnson, E.J.T. Burns, J.P. Quintenz, K.W. Bieg, A.V. Farnsworth Jr., L.P. Mix and M.A. Palmer, J. Appl. Phys. 52, 168 (1981).

5. "Kinetic Stability Theorem for Relativistic Nonneutral Electron Flow in a Planar Diode With Applied Magnetic Field," R.C. Davidson, submitted for publication (1983).

6. "Nonlinear Bound on Unstable Electrostatic Fluctuation Energy for Nonrelativistic Nonneutral Electron Flow in a Planar Diode with Applied Magnetic Field," R.C. Davidson, submitted for publication (1983).

7. R.C. Davidson and H.S. Uhm, Phys. Fluids 25, 2089 (1982).

8. T.M. O’Neil, Phys. Fluids 23, 2216 (1980).

9. R.C. Davidson, S.M. Mahajan and H.S. Uhm, Phys. Fluids 19, 1608 (1976).

10. R.C. Davidson, Theory of Nonneutral Plasmas (Benjamin, Reading, Mass., 1974) pp. 155-177.

11. H.V. Wong, M.L. Sloan, J.R. Thompson and A.T. Drobot, Phys. Fluids 16, 902 (1973).

12. D.A. Hammer and N. Rostoker, Phys. Fluids 13, 1831 (1970).

13. R.C. Davidson and N.A. Krall, Phys. Fluids 13,1543 (1970).

14. cf. Ref. 10, pp. 17-89.

15. J. Swegle and E. Ott, Phys. Fluids 24, 1821 (1981).

16. J. Swegle and E. Ott, Phys. Rev. Lett. 46, 929 (1981).

17. C.A. Kapetanakos, D.A. Hammer, C.D. Striffler and R.C. Davidson, Phys. Rev. Lett. 30, 1303 (1973).

18. B.L. Bogema and R.C. Davidson, Phys. Fluids 14, 1436 (1971).

19. R.J. Briggs, J.D. Daugherty and R.H. Levy, Phys. Fluids 13, 421 (1970).

20. R.H. Levy, Phys. Fluids 8, 1288 (1965).

21. "Macroscopic Extraordinary-Mode Stability Properties of Relativistic Nonneutral Electron Flow in a Planar Diode With Applied Magnetic Field," R.C. Davidson and K. Tsang, submitted for publication (1983). 


\section{FIGURE CAPTIONS}

1. Cylindrical diode configuration with cathode at $r=a$ and anode at $r=b$, and applied axial magnetic field $B_{0}(x)=B_{0} \hat{e}_{z}$. Equilibrium electron flow is in the $\theta$-direction.

2. (a) Rectangular density profile $n_{b}^{0}(r)$ assumed in Eq. (18). (b) Corresponding angular velocity profile $\omega_{b}^{-}(r)$ in Eq. (19).

3. Class of equilibrium density profiles $n_{b}^{0}(r)$ that are (a) stable [Eq. (55)], (b) unstable [Eq. (61)], and (c) unstable [Eq. (61)], for low-frequency flute perturbations satisfying Egs. (44)-(46).

4. Hollow electron density profile that exhibits strong diocotron instability (R.C. Davidson, Theory of Nonneutral Plasmas, Benjamin, Reading, Mass., 1974).

5. Rectangular density profile $n_{b}^{0}(r)$ assumed in stability analysis in Sec. VI.A. A large-aspect-ratio diode with $a \gg \Delta_{u}, \Delta_{v}$ is assumed.

6. Density profile $n_{b}^{0}(r)$ with density bump that leads to the resonant diocotron instability discussed in Sec. VI.B.

7. Tables of Rew/ $/ \omega_{c}$ and $I m w / \omega_{c}$ versus $\ell$ [Eq. (125)] for $b / a=3, r_{b} / a=2$, and (a) $s=0.5$, and (b) $B=0.2$, for the rectangular density profile in Eq. (127).

8. Plot of $n_{b}^{0}(r)$ versus $r$ for the bell-shaped density profile in Eq. (128).

9. Table of Rew $/ \omega_{c}$ and $I m \omega / \omega_{c}$ versus $s=4 \pi \hat{n}_{b} m c^{2} / B_{0}^{2}$ [Eq. (125)] for $b / a=3, r_{b} / a=2$ and $\ell=2$ for the bell-shaped density profile in Eq. (128).

10. (a) Plots of $n_{b}^{0}(r) / \hat{n}_{b}$ and $\omega_{b}^{-}(r) / \omega_{c}$ versus $r / a$ for the rectangular density profile in Eq. (127) with $r_{b} / a=2, s=0.5$ and $b / a=3$ [case presented in Fig. 7(a)]; (b) Plot of $\left[\omega_{r}-\ell \omega_{b}^{-}(r)\right] / \omega_{c}$ versus $r / a$ obtained from Eq. (125) for $\ell=4$ and $s=0.5$; (c) Plots of $R e \delta \phi^{\ell}$ and $I m \delta \phi^{\ell}$ versus $r / a$ obtained from Eq. (125) for $\ell=4$ and $s=0.5$.

11. (a) Plots of $n_{b}^{0}(r) / \tilde{n}_{b}$ and $\omega_{b}^{-}(r) / \omega_{c}$ versus $r / a$ for the bell-shaped density profile in Eq. (128) with $r_{b} / a=2, s=1.0$, and $b / a=3$ (case presented in Fig. 9); (b) Plot of $\left[\omega_{r}-\ell \omega_{b}^{-}(r)\right] / \omega_{c}$ versus $r / a$ obtained from Eq. (125) for $\ell=2$ and $s=1.0$; (c) Plots of $R e \delta \phi^{\ell}$ and $I m \delta \phi^{\ell}$ versus $r / a$ obtained from Eq. (125) for $\ell=2$ and $s=1.0$.

12. Tables of Rew/ $/ \omega_{c}$ and $I m w / \omega_{c}$ versus $\ell$ [Eq. (125)] for $b / a=3, r_{b}^{+} / a=2, r_{b}^{-} / a=7 / 5$, and (a) $s=0.5$, and (b) $s=0.2$, for the hollow density profile in Eq. (131). 
13. (a) Plots of $n_{b}^{0}(r) / \hat{n}_{b}$ and $\omega_{b}^{-}(r) / \omega_{c}$ versus $r / a$ for the hollow density profile in Eq. (131) with $r_{b}^{-} / a=$ $7 / 5, r_{b}^{+} / a=2, b / a=3$ and $s=0.5$ [case presented in Fig. 12(a)]; (b) Plot of $\left[\omega_{r}-\ell \omega_{b}^{-}(r)\right] / \omega_{c}$ versus $r / a$ obtained from Eq. (125) for $\ell=2$ and $s=0.5$; (c) Plots of Re $\delta \phi^{\ell}$ and $I m \delta \phi^{\ell}$ versus $r / a$ obtained from Eq. (125) for $\ell=2$ and $s=0.5$. 


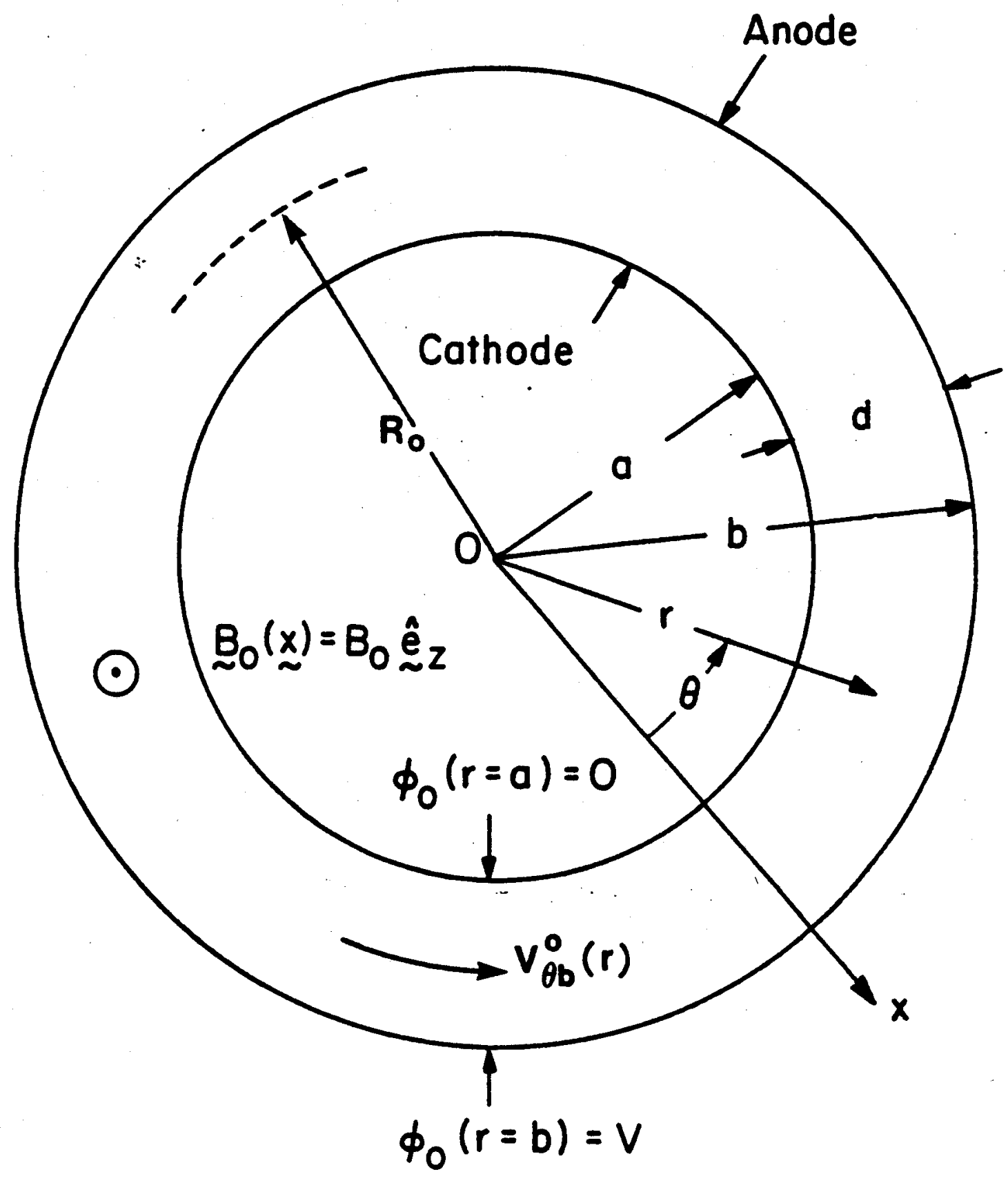

Fig. 1: Cylindrical diode configuration with cathode at $r=a$ and anode at $r=b$, and applied axial magnetic field $B_{0}(x)=B_{0} \hat{e}_{z}$. Equilibrium electron flow is in the $\theta$-direction. 

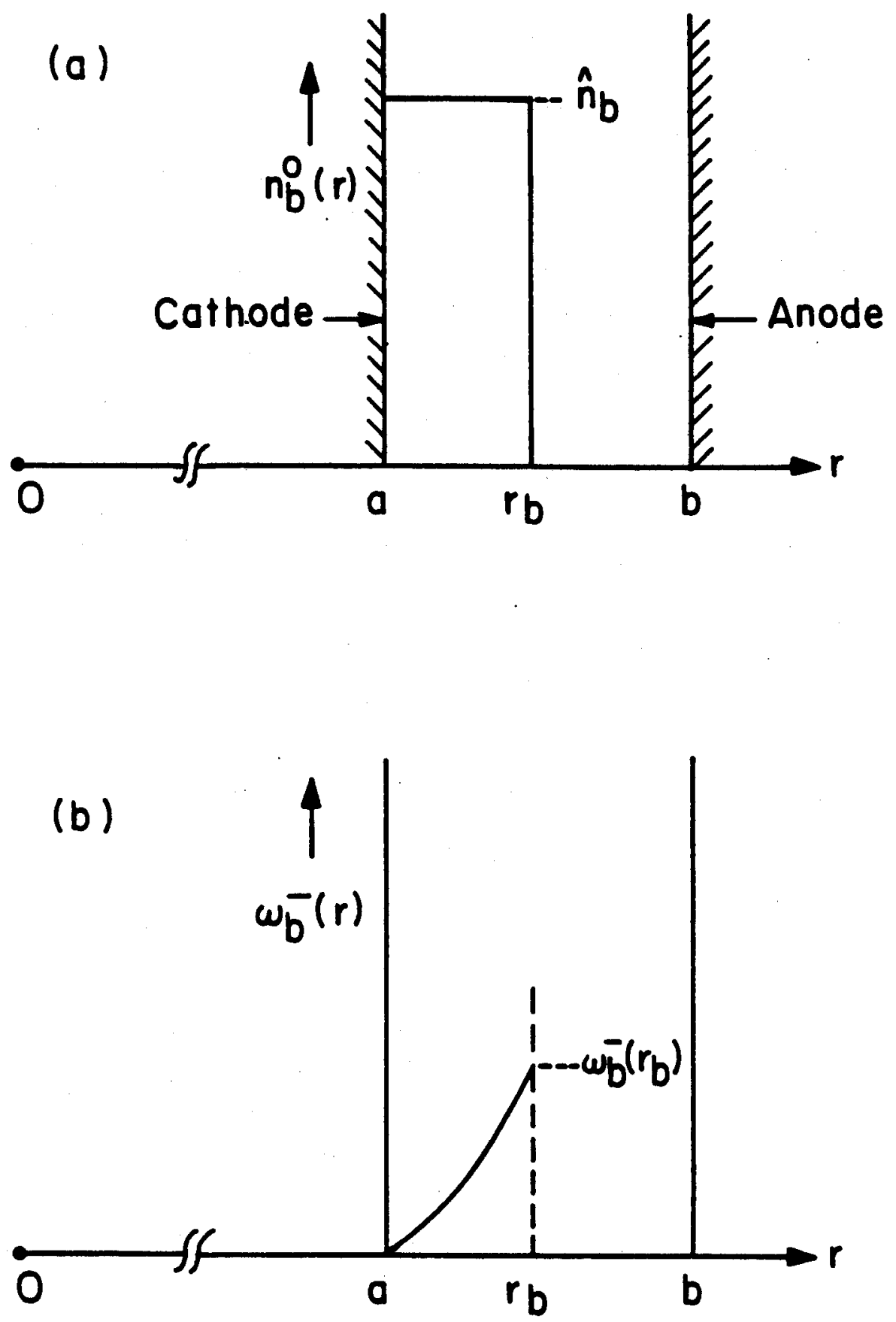

Fig. 2: (a) Rectangular density profile $n_{b}^{0}(r)$ assumed in Eq. (18). (b) Corresponding angular velocity profile $\omega_{b}^{-}(r)$ in Eq. (19). 

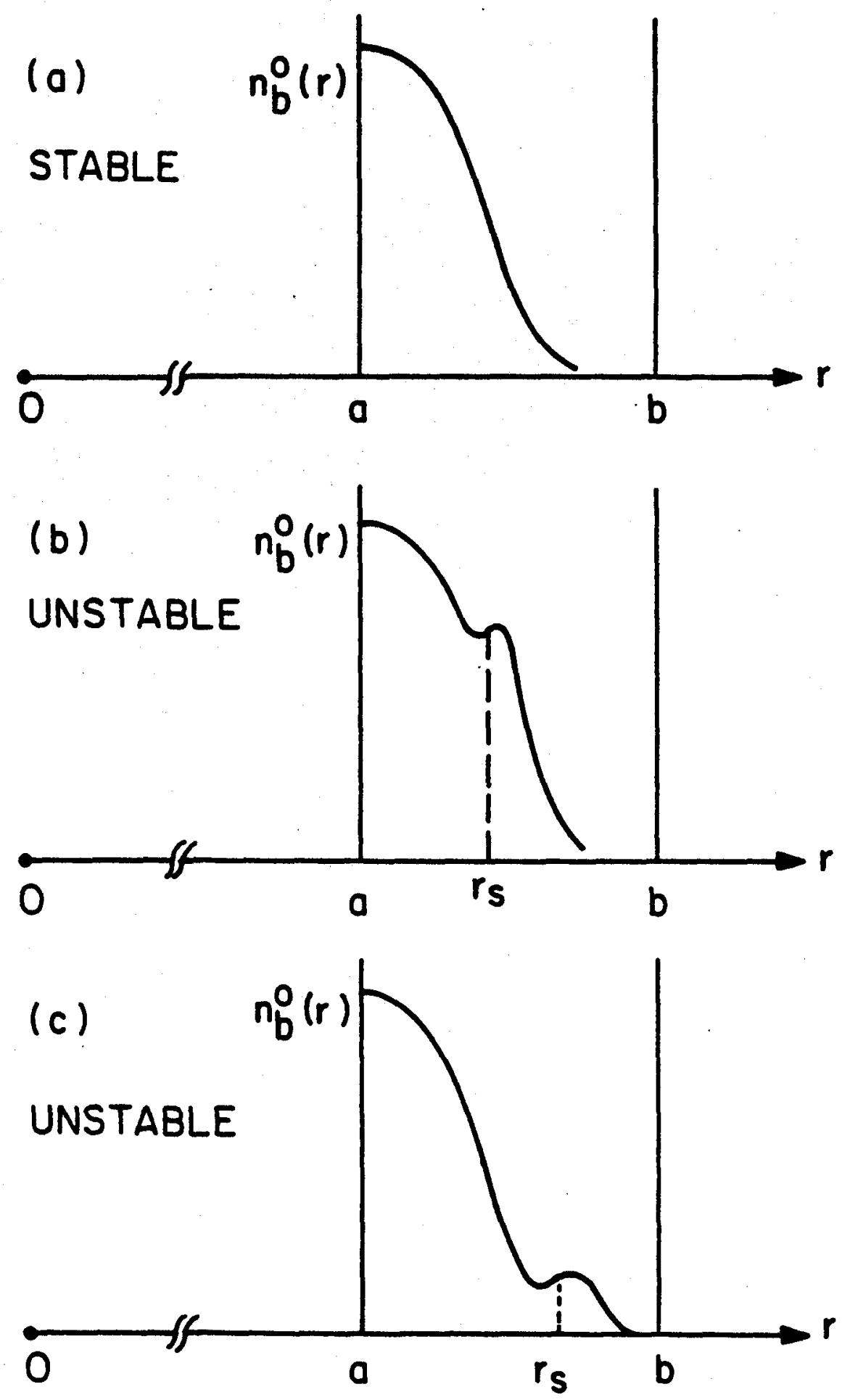

Fig. 3: Class of equilibrium density profiles $n_{b}^{0}(r)$ that are (a) stable [Eq. (55)], (b) unstable [Eq. (61)], and (c)unstable [Eq. (61)], for low-frequency flute perturbations satisfying Eqs. (44)-(46). 


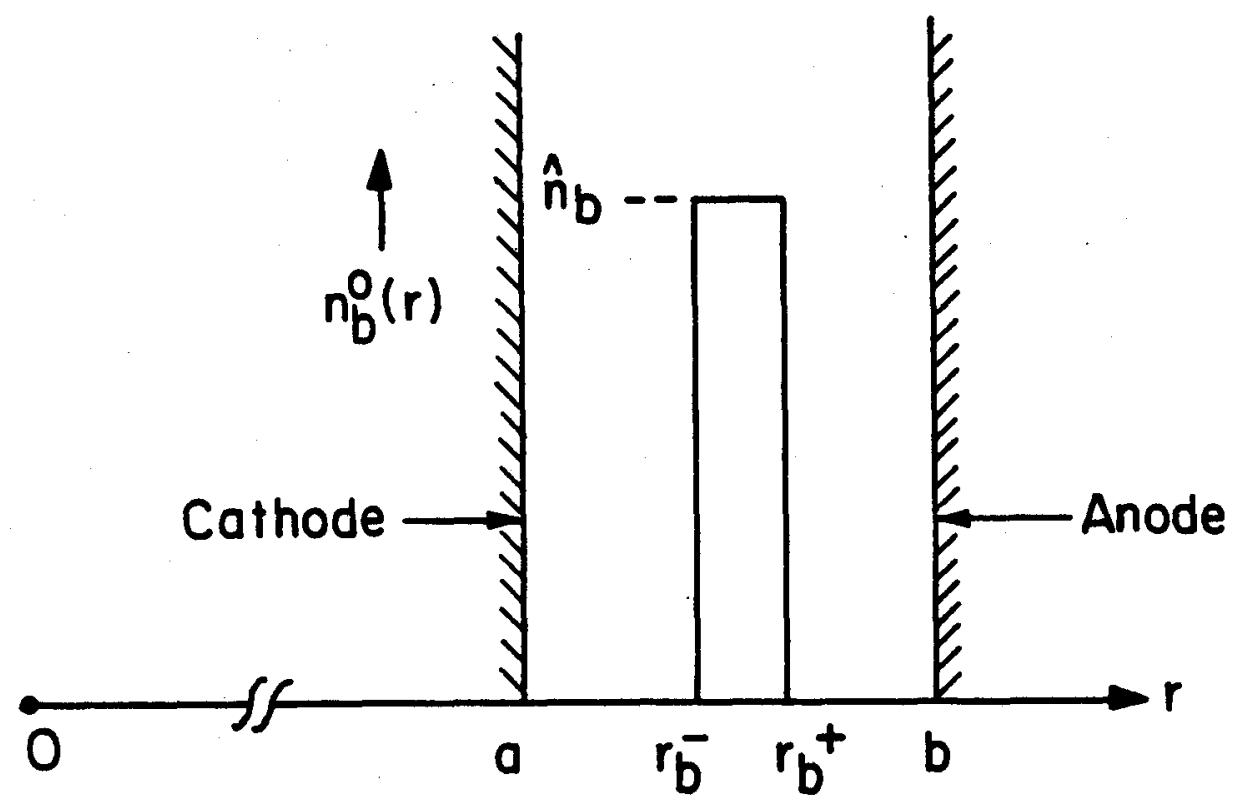

Fig. 4: Hollow electron density profile that exhibits strong diocotron instability (R.C. Davidson. Theory of Nonneutral Plasmas, Benjamin, Reading, Mass., 1974).

40 


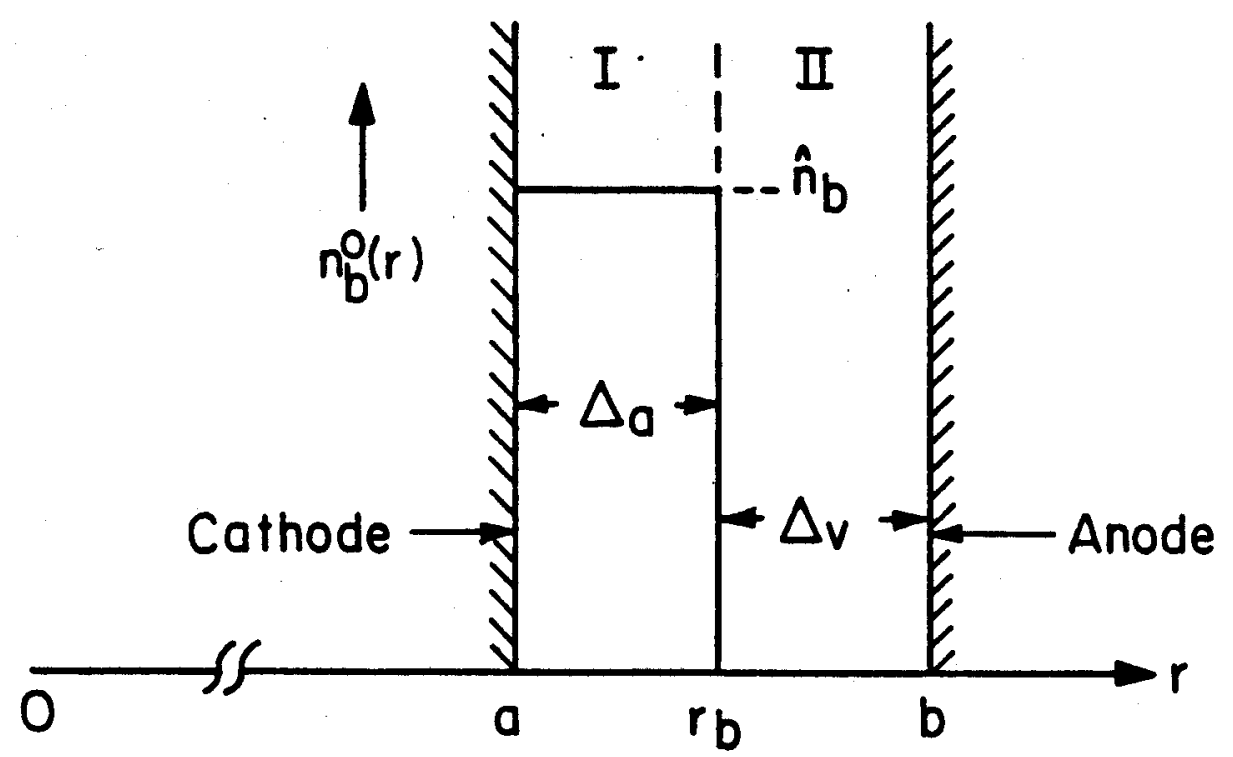

Fig. 5: Rectangular density profile $n_{b}^{0}(r)$ assumed in stability analysis in Sec. VI.A. A large-aspect-ratio diode with $a \gg \Delta_{a}, \Delta_{v}$ is assumed. 


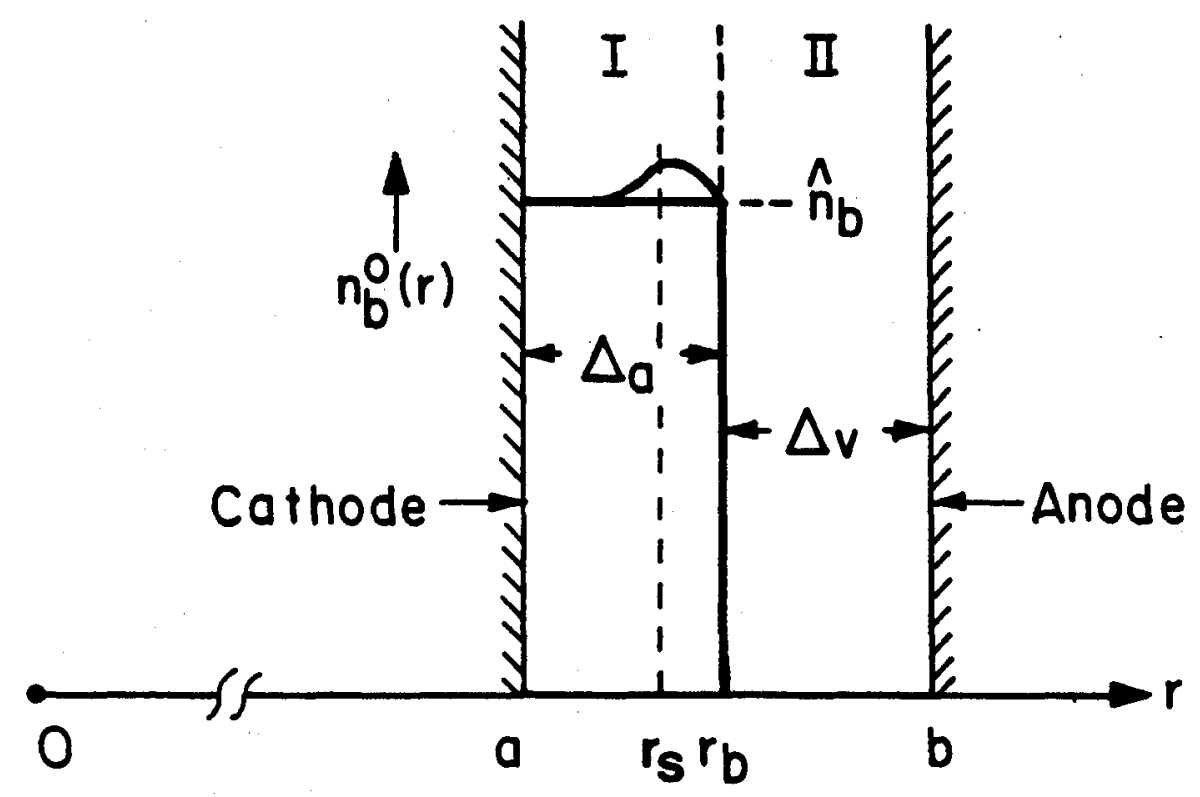

Fig. 6: Density profile $n_{b}^{0}(r)$ with density bump that leads to the resonant diocotron instability discussed in Sec. VI.B. 


\begin{tabular}{|c|c|c|}
\hline \multicolumn{3}{|c|}{ (a): $\delta=\omega_{p b}^{2} / \omega_{c}^{2}=0.5$} \\
\hline$\ell$ & Rew $/ \omega_{c}$ & $I \mathrm{mw} / \omega_{c}$ \\
\hline 1 & 0.066 & $0.59 \times 10^{-2}$ \\
\hline 2 & 0.209 & $1.32 \times 10^{-2}$ \\
\hline 3 & 0.417 & $2.35 \times 10^{-2}$ \\
\hline 4 & 0.658 & $2.44 \times 10^{-2}$ \\
\hline 5 & 0.867 & $0.26 \times 10^{-2}$ \\
\hline
\end{tabular}

\begin{tabular}{|c|c|c|}
\hline \multicolumn{3}{|c|}{ (b): $s=\omega_{p b}^{2} / \omega_{c}^{2}=0.2$} \\
\hline$\ell$ & Rew/ $/ \omega_{c}$ & $I \mathrm{mw} / \omega_{c}$ \\
\hline 1 & 0.028 & $1.65 \times 10^{-4}$ \\
\hline 2 & 0.077 & $2.79 \times 10^{-4}$ \\
\hline 3 & 0.143 & $2.49 \times 10^{-4}$ \\
\hline 4 & 0.219 & $1.76 \times 10^{-4}$ \\
\hline 5 & 0.298 & $1.22 \times 10^{-4}$ \\
\hline
\end{tabular}

Fig. 7: Tables of Rew/ $\omega_{c}$ and $I m w / \omega_{c}$ versus $\ell$ [Eq. (125)] for $b / a=3$, $r_{b} / a=2$, and (a) $s=0.5$, and (b) $s=0.2$, for the rectangular density profile in Eq. (127). 


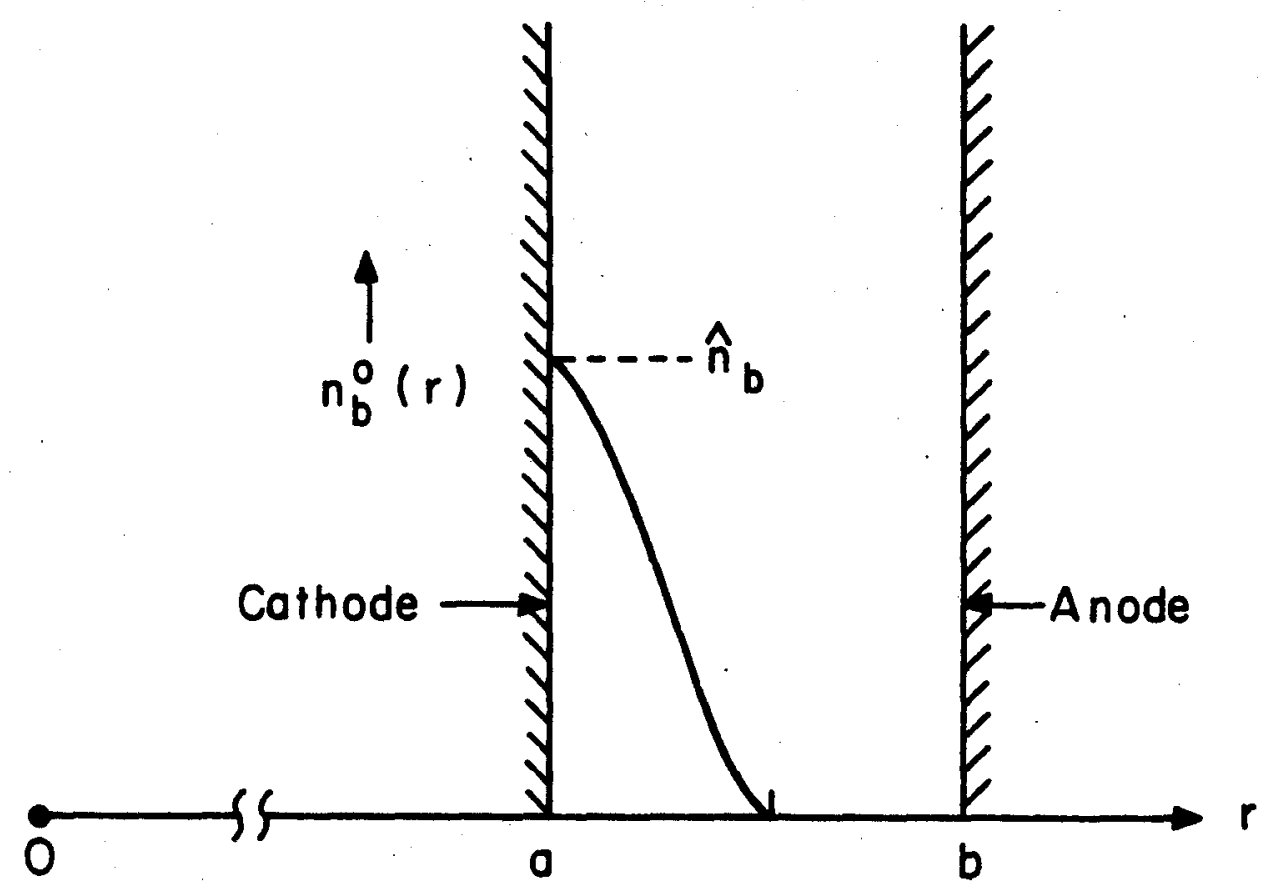

Fig. 8: Plot of $n_{b}^{0}(r)$ versus $r$ for the bell-shaped density profile in Eq. (128). 


\begin{tabular}{|c|c|c|}
\hline \multicolumn{3}{|c|}{$\ell=2 ; b / a=3 ; \quad r_{b} / a=2$} \\
\hline$\delta$ & $R e w / \omega_{c}$ & $I m w / \omega_{c}$ \\
\hline 0.48 & 0.074 & $0.005 \times 10^{-1}$ \\
\hline 0.5 & 0.077 & $0.012 \times 10^{-1}$ \\
\hline 0.6 & 0.089 & $0.067 \times 10^{-1}$ \\
\hline 0.7 & 0.101 & $0.178 \times 10^{-1}$ \\
\hline 0.8 & 0.117 & $0.397 \times 10^{-1}$ \\
\hline 0.9 & 0.156 & $0.779 \times 10^{-1}$ \\
\hline 1.0 & 0.248 & $0.977 \times 10^{-1}$ \\
\hline
\end{tabular}

Fig. 9: Table of $R \omega^{\prime} / \omega_{c}$ and $I m w / \omega_{c}$ versus $s=4 \pi \hat{n}_{b} m c^{2} / B_{0}^{2}$ [Eq. (125)] for $b / a=3, r_{b} / a=2$, and $\ell=2$ for the bell-shaped density profile in Eq. (128). 


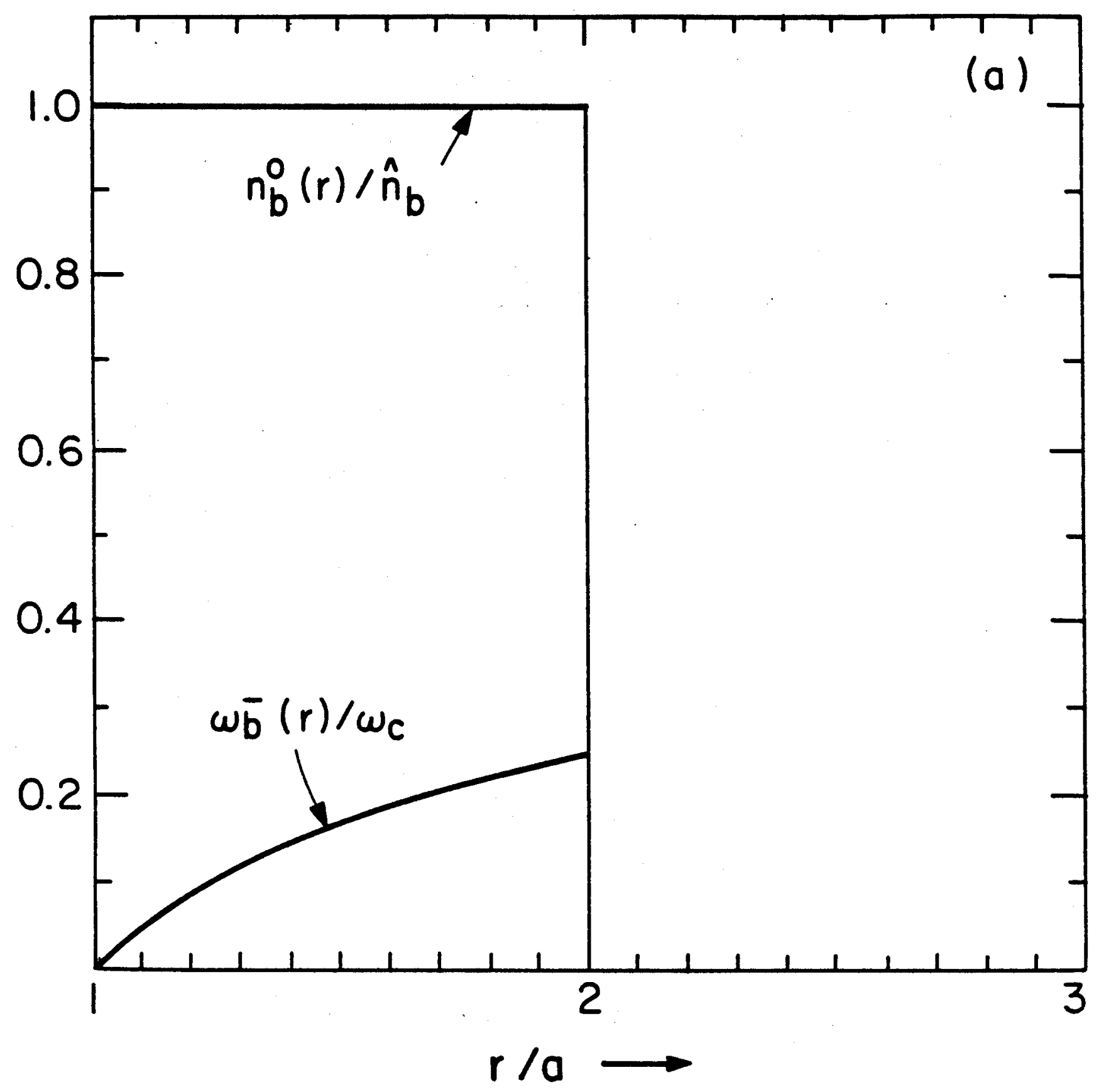

Fig. 10(a): Plots of $n_{b}^{0}(r) / \hat{n}_{b}$ and $\omega_{b}^{-}(r) / \omega_{c}$ versus $r / a$ for the rectangular density profile in Eq. (127) with $r_{b} / a=2,8=0.5$ and $b / a=3$ [case presented in Fig. 7(a)]. 


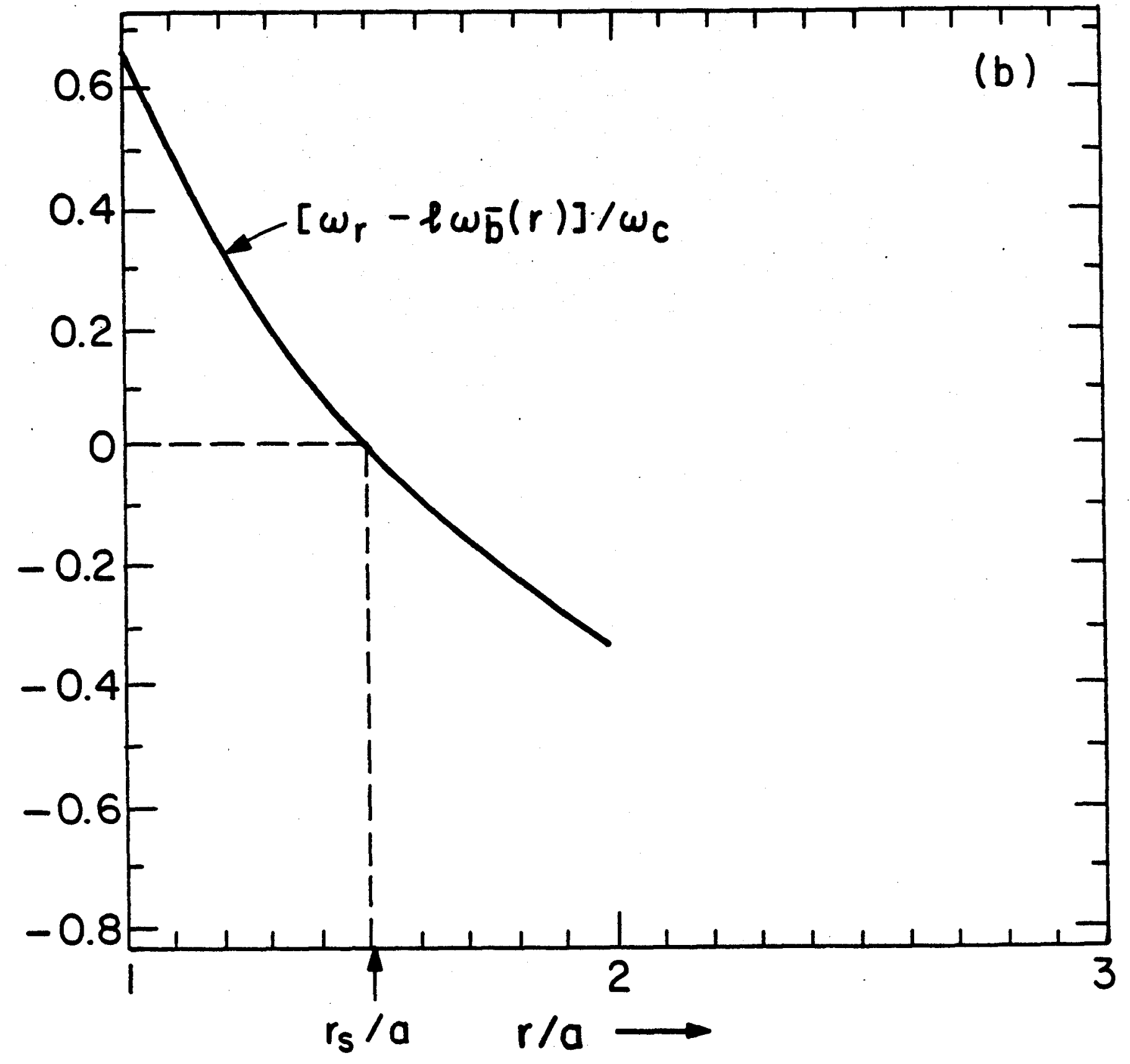

Fig. 10(b): Plot of $\left[\omega_{r}-\ell \omega_{b}-(r)\right] / \omega_{c}$ versus $r / a$ obtained from Eq. (125) for $\ell=4$ and $s=0.5$ for the rectangular density profile in Eq. (127). 


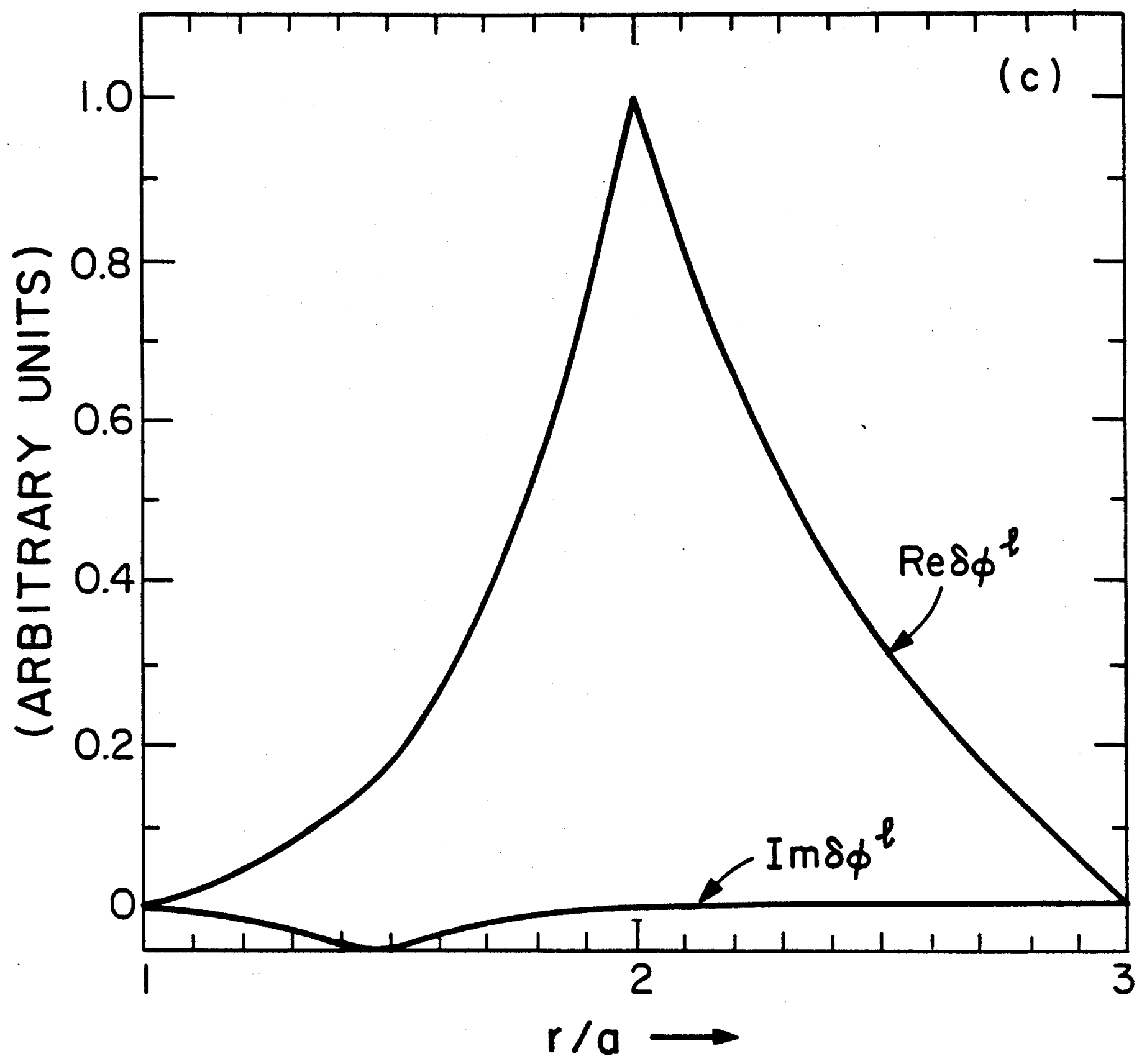

Fig. 10(c): Plots of Re $\delta \phi^{\ell}$ and $I m \delta \phi^{\ell}$ versus $r / a$ obtained from Eq. (125) for $\ell=4$ and $s=0.5$ for the rectangular density profile in Eq. (127). 


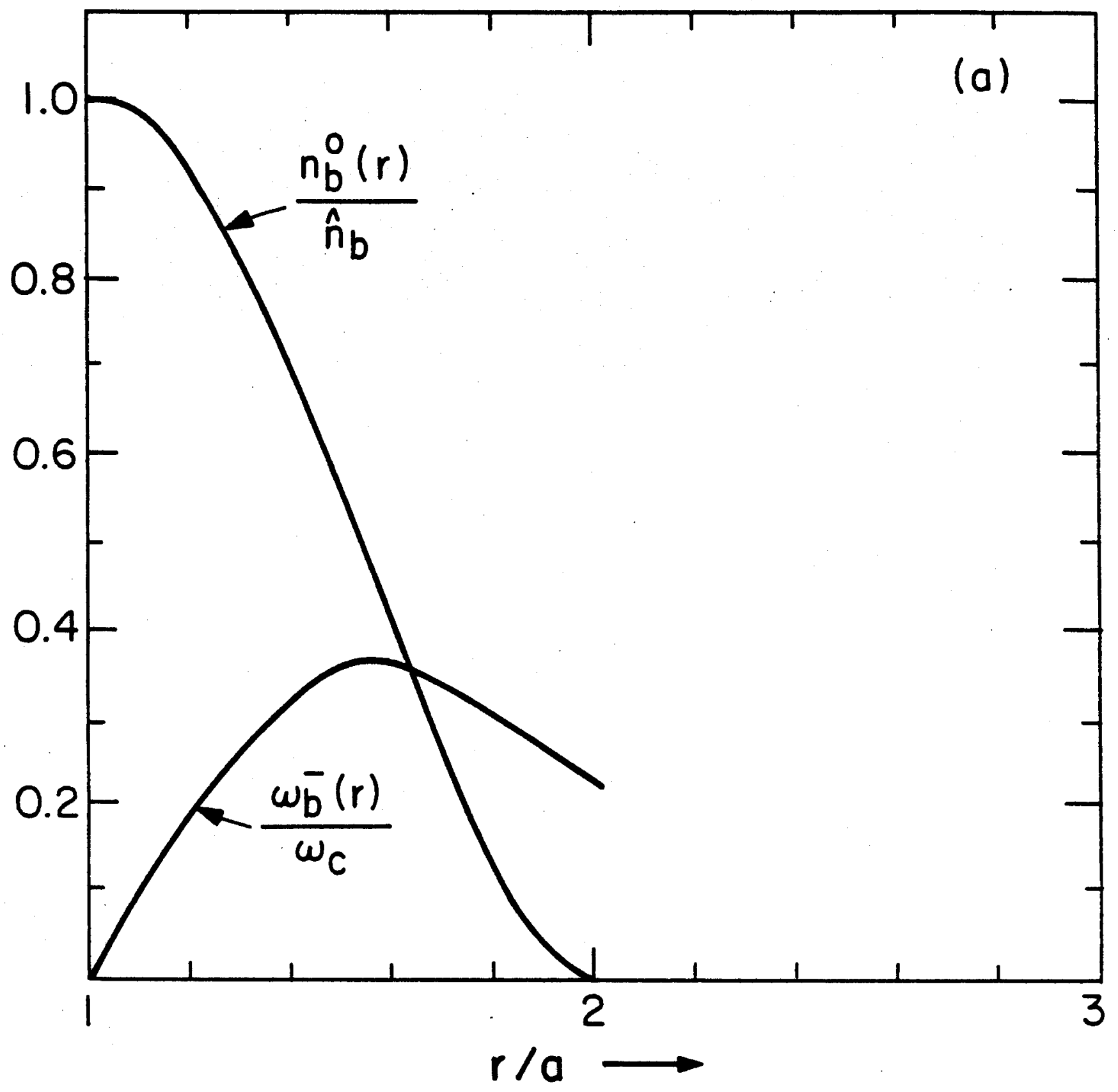

Fig. 11(a): Plots of $n_{b}^{0}(r) / \hat{n}_{b}$ and $\omega_{b}^{-}(r) / \omega_{c}$ versus $r / a$ for the bell-shaped density profile in Eq. (128) with $r_{b} / a=2,8=1.0$, and $b / a=3$. (case presented in Fig. 9). 


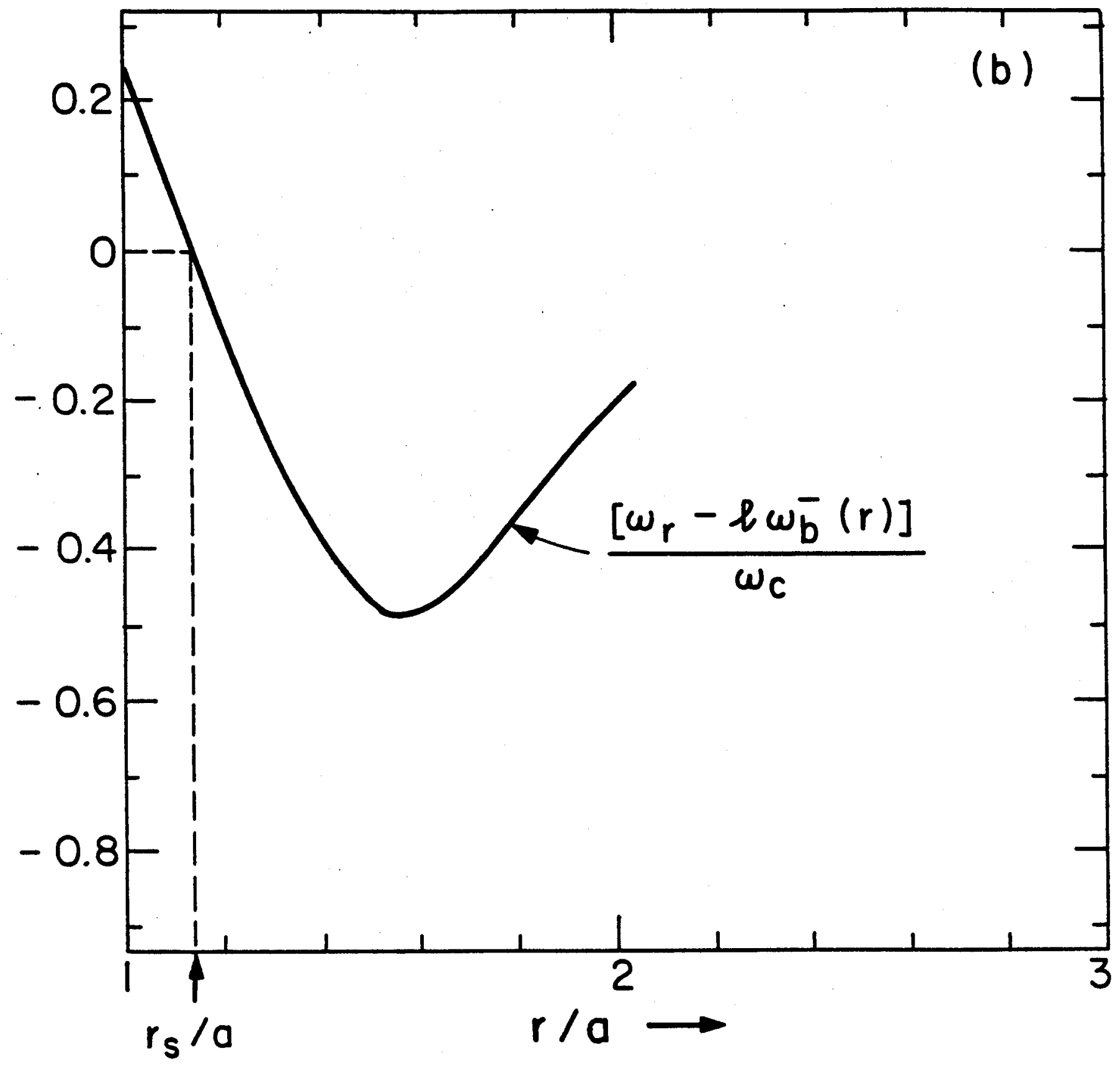

Fig. 11(b): Plot of $\left[\omega_{r}-\ell \omega_{b}^{-}(r)\right] / \omega_{c}$ versus $r / a$ obtained from Eq. (125) for $\ell=2$ and $s=1.0$ for the bell-shaped density profile in Eq. (128). 


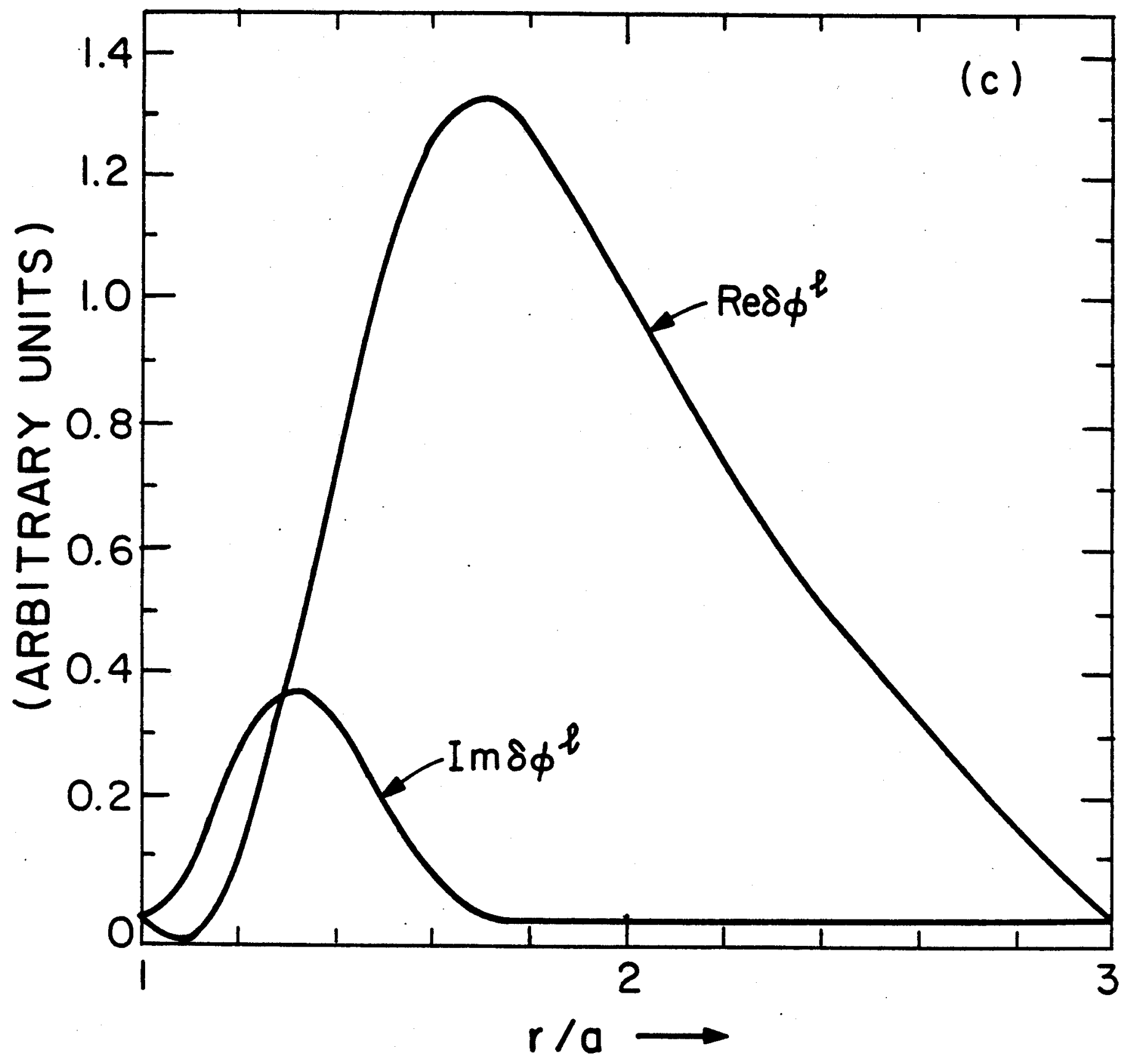

Fig. 11(c): Plots of $R e \delta \phi^{\ell}$ and $I m \delta \phi^{\ell}$ versus $r / a$ obtained from Eq. (125) for $\ell=2$ and $s=1.0$ for the bell-shaped density profile in Eq. (128). 


\begin{tabular}{|c|c|c|}
\hline \multicolumn{3}{|c|}{ (a): $\quad s=4 \pi \tilde{n}_{b} m c^{2} / B_{0}^{2}=0.5$} \\
\hline$\ell$ & Rew/的 & $\operatorname{Im} \omega / \omega_{c}$ \\
\hline$\overline{1}$ & 0.069 & $0.25 \times 10^{-1}$ \\
\hline 2 & 0.144 & $0.59 \times 10^{-1}$ \\
\hline 3 & 0.221 & $0.84 \times 10^{-1}$ \\
\hline 4 & 0.301 & $0.86 \times 10^{-1}$ \\
\hline 5 & 0.400 & $0.45 \times 10^{-1}$ \\
\hline
\end{tabular}

\begin{tabular}{|c|c|c|}
\hline \multicolumn{3}{|c|}{ (b): $s=4 \pi \hat{\hbar}_{b} m c^{2} / B_{0}^{2}=0.2$} \\
\hline$\ell$ & $R e w / \omega_{c}$ & $I m \omega / \omega_{c}$ \\
\hline 1 & 0.025 & $0.095 \times 10^{-1}$ \\
\hline 2 & 0.051 & $0.206 \times 10^{-1}$ \\
\hline 3 & 0.079 & $0.282 \times 10^{-1}$ \\
\hline 4 & 0.107 & $0.266 \times 10^{-1}$ \\
\hline 5 & 0.151 & $0.013 \times 10^{-1}$ \\
\hline
\end{tabular}

Fig. 12: Tables of Rew/ $/ \omega_{c}$ and $I m w / \omega_{c}$ versus $\ell$ [Eq. (125)] for $b / a=3$, $r_{b}^{+} / a=2, r_{b}^{-} / a=7 / 5$, and (a) $s=0.5$, and (b) $s=0.2$, for the hollow density profile in Eq. (131). 


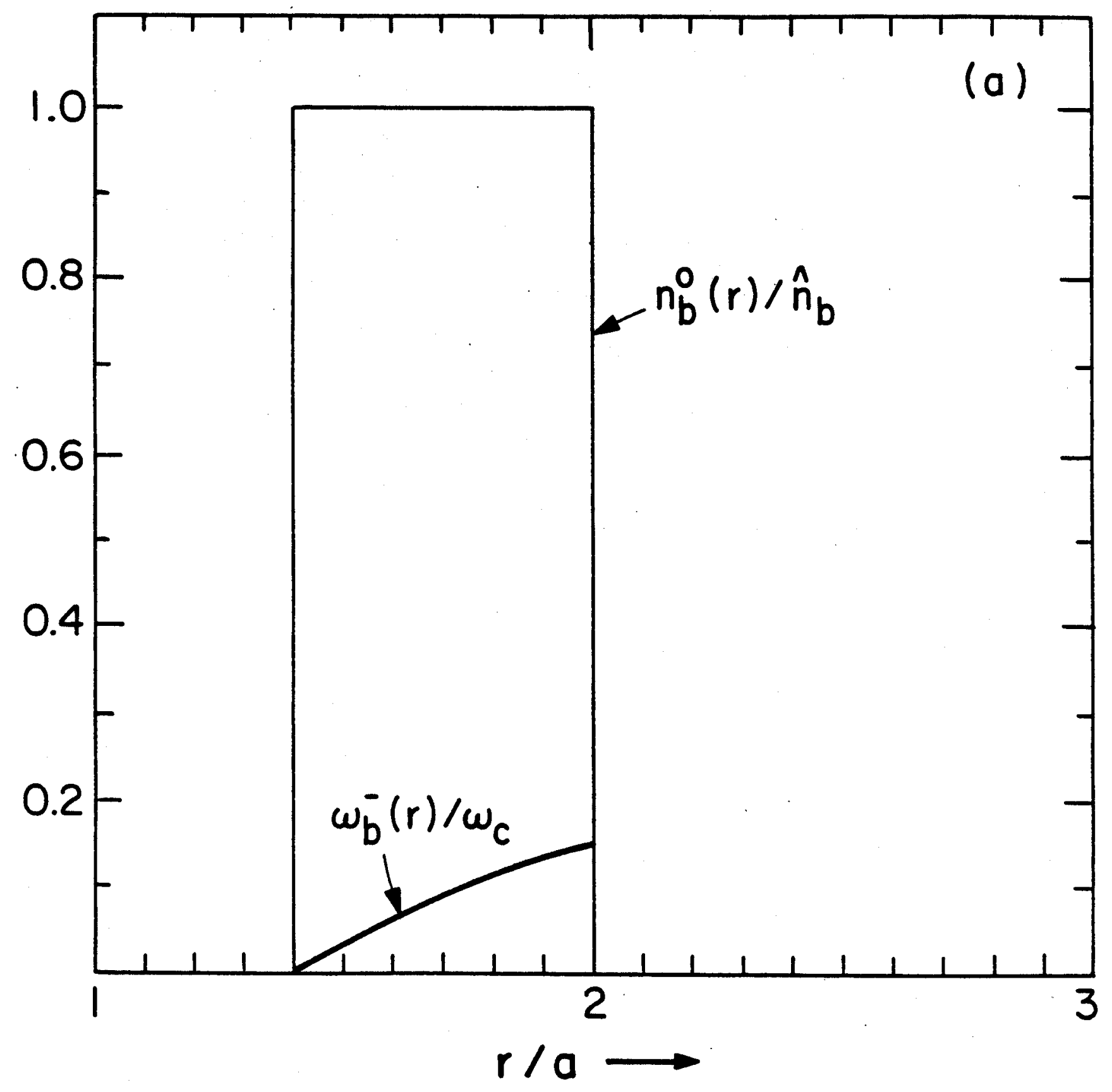

Fig. 13(a): Plots of $n_{b}^{0}(r) / \hat{n}_{b}$ and $\omega_{b}^{-}(r) / \omega_{c}$ versus $r / a$ for the hollow density profile in Eq. (131) with $r_{b}^{-} / a=7 / 5, r_{b}^{+} / a=2, b / a=3$ and $s=0.5$ [case presented in Fig. 12(a)]. 


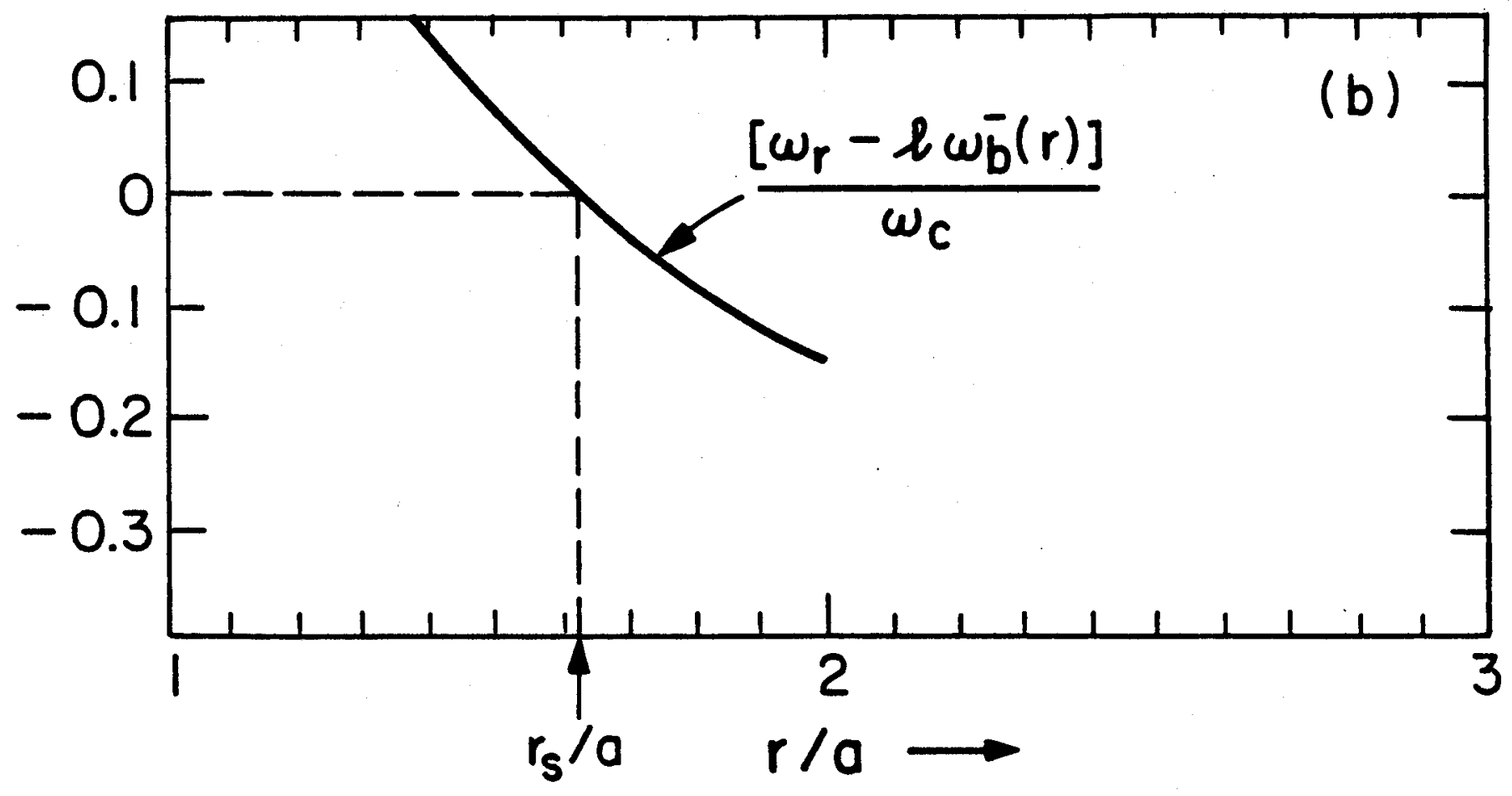

Fig. 13(b): Plot of $\left[\omega_{r}-\ell \omega_{b}^{-}(r)\right] / \omega_{c}$ versus $r / a$ obtained from Eq. (125) for $\ell=2$ and $s=0.5$ for the hollow density profile in Eq. (131). 


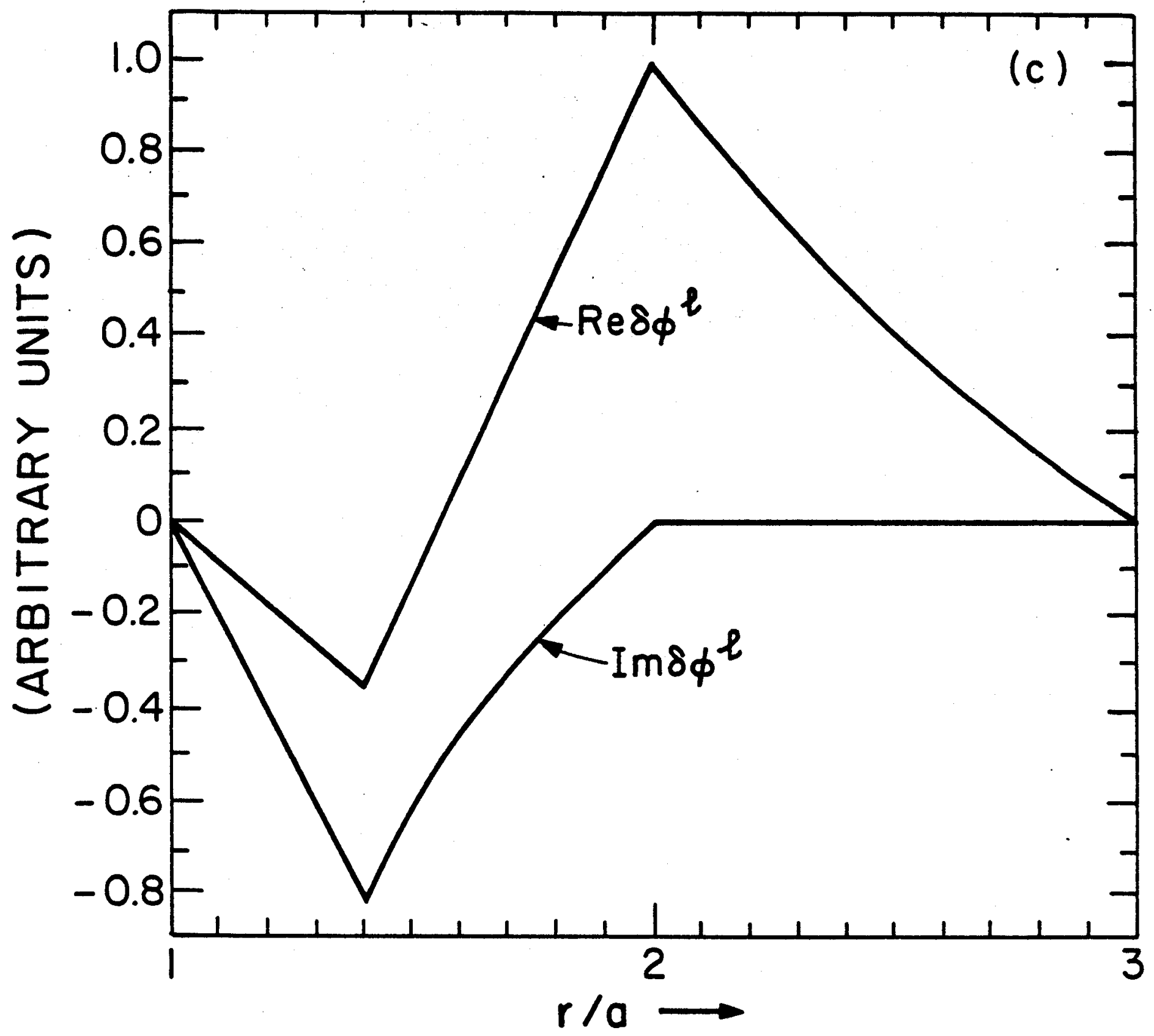

Fig. 13(c): Plots of $R e \delta \phi^{\ell}$ and $I m \delta \phi^{\ell}$ versus $r / a$ obtained from Eq. (125) for $\ell=2$ and $s=0.5$ for the hollow density profile in Eq. (131). 